The Astrophysical Journal Supplement Series, 176:262-275, 2008 May

(C) 2008. The American Astronomical Society. All rights reserved. Printed in U.S.A.

\title{
COUSINS PHOTOMETRY AND TEMPERATURES FOR THE HYADES, COMA, NGC 752, PRAESEPE, AND M67
}

\author{
B. J. TAYLOR AND M. D. JONER ${ }^{1}$ \\ Department of Physics and Astronomy, N283 ESC, Brigham Young University, Provo, UT 84602-4360 \\ AND \\ E. J. JEFFERY \\ Astronomy Department, University of Texas, Austin, TX 78712 \\ Received 2007 October 20; accepted 2007 November 9
}

\begin{abstract}
In this paper, new Cousins VRI data are presented for NGC 752 and Praesepe, and new and extant data are combined into an augmented database for M67. For those three clusters, catalogs containing Cousins VRI photometry, reddening-corrected values of $(V-K)_{\mathrm{J}}$, and temperatures are produced. The same is done for Coma by using both previously published and newly derived Cousins photometry. An extant set of catalogs for the Hyades is updated to include $V$ magnitudes and values of $(R-I)_{\mathrm{C}}$ that were published after the original catalogs appeared. Finally, M67 $V$ magnitudes published previously by Sandquist are corrected for an effect that depends on location on the face of the cluster. The corrected data and values of $(V-I)_{\mathrm{C}}$ given by Sandquist are then set out in a supplementary catalog. Data files containing all of these catalogs are deposited in the CDS archives. To assess the quality of the data in the catalogs, the consistency of extant Cousins VRI databases is tested by performing analyses with the following features: (1) quantities as small as a few millimags are regarded as meaningful; (2) statistical analysis is applied; (3) no use is made of data other than VRI measurements and comparable results; (4) no inferences are drawn from colormagnitude comparisons; (5) pertinent data that have not been included previously are analyzed; and (6) results based on direct comparisons of stellar groups at the telescope are featured. In this way, it is found that our updated M67 color data and those of Sandquist are on the E region zero point. In contrast, values of $(V-I)_{\mathrm{C}}$ from Montgomery and collaborators are found to be too red by $27 \pm 3 \mathrm{mmag}$, with an even larger offset being likely for unpublished data from Richer and his collaborators. Zero-point tests of our Cousins VRI colors for Coma, Praesepe, and NGC 752 are also satisfactory. Scale factor tests of the M67 colors are performed, and a likely scale factor error in the Montgomery et al. colors is found. However, it appears at present that the scale factors of our M67 colors and those of Sandquist are satisfactory. For the most part, zero-point tests of the assembled $V$ magnitudes are also satisfactory, although it is found that further work on the $V$ magnitudes for Praesepe and M67 would be useful. To put these results in perspective, it is pointed out that photometric tests that are satisfactory at the few-millimag level have been published for some two decades and so are not appearing for the first time in this paper.
\end{abstract}

Subject headings: Hertzsprung-Russell diagram - open clusters and associations: individual (Coma, Hyades, M67, NGC 752, Praesepe) - stars: fundamental parameters

\section{INTRODUCTION}

Two years ago, Taylor \& Joner (2005, hereafter TJ05) published three catalogs containing temperatures and values of $(R-$ $I)_{\mathrm{C}}$ for the Hyades. In this followup paper, those catalogs are updated and counterparts are given for four additional clusters: Coma, NGC 752, Praesepe, and M67. Reliable reddening values are available for all five clusters, and precise values of $[\mathrm{Fe} / \mathrm{H}]$ are known for all of them except NGC 752 (Taylor 2006, 2007a, $2007 \mathrm{~b}){ }^{2}$ One major aim of this paper is to fulfill the remaining requirements for high-quality color-magnitude analyses of these clusters.

For NGC 752 and Praesepe, new Cousins VRI photometry is presented here. For M67, previously published photometry is combined with new results to form an expanded Cousins VRI database (to be called the "augmented" M67 database below). Statistical

\footnotetext{
1 Visiting astronomer, Kitt Peak National Observatory and Cerro Tololo InterAmerican Observatory, National Optical Astronomy Observatories, which are operated by the Association of Universities for Research in Astronomy, Inc., under contract with the National Science Foundation.

2 After first citations, papers by Taylor \& Joner and Joner \& Taylor will be cited as "TJ" and "JT," respectively, with the last two digits of the publication year added. "T" followed by two digits represents a paper published by Taylor. All of these abbreviations are given in the reference list.
}

tests of zero-point accuracy are then performed on the data for all three clusters. In addition, such tests are applied to previously published VRI photometry for Coma and M67. Particular attention is given to the M67 tests because there appear to be zero-point differences among some extant M67 VRI photometry. Zero points that are deemed to be fully reliable will be established in response.

The plan for this paper is as follows. In $\S 2$ a description of the sources and reduction techniques for the new data is given. The M67 zero-point problem is stated in full in $\S 3$, and the first basic steps toward its solution are taken. In $\S 4$ the adopted zero-point analysis technique is set out in detail. Results of the analysis are given for M67 in $\S \S 5-7$ and for Coma, Praesepe, and NGC 752 in $\S 8$. The augmented M67 data also include $V$ magnitudes, so the results of zero-point tests of those data are reported in $\S 9$. For both the four clusters just mentioned and the Hyades, the new and revised catalogs and the procedure used to construct them are described in $\S 10$. In $\S 11$ essential perspectives on literature practice are given and recommendations for future improvements are made. The paper concludes with a summary in $\S 12$.

\section{NEW DATABASES: SOURCES AND REDUCTIONS}

The new photometry can be grouped into three databases. One of them includes photomultiplier data measured from 1972 
TABLE 1

M67 Photometry: Formal Positional Corrections

\begin{tabular}{|c|c|c|c|c|}
\hline Source & Color Index ${ }^{\mathrm{a}}$ & $C_{\alpha}{ }^{\mathrm{b}}$ & $C_{\delta}^{\mathrm{b}}$ & $C_{0}{ }^{\mathrm{c}}$ \\
\hline CTIO (this paper) & $(R-I)_{\mathrm{C}}$ & $-0.6 \pm 1.0$ & $2.9 \pm 0.8$ & 0 \\
\hline KPNO (this paper)..... & $(V-R)_{\mathrm{L}}$ & $1.5 \pm 0.2$ & $1.7 \pm 0.3$ & 0 \\
\hline Laugalys et al. (2004) ${ }^{\mathrm{d}}$. & $(V-I)_{\mathrm{C}}$ & $-0.8 \pm 0.2$ & 0 & $2.6 \pm 0.9$ \\
\hline 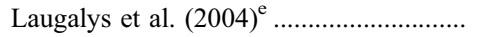 & $V$ & $-0.5 \pm 0.2$ & $-0.6 \pm 0.2$ & $24 \pm 1$ \\
\hline Montgomery et al. (1993) ......................... & $V$ & $2.8 \pm 0.6$ & $0.3 \pm 0.6$ & $-12.9 \pm 2.6$ \\
\hline Sandquist (2004) ............ & $V$ & $2.4 \pm 0.3$ & $-2.8 \pm 0.4$ & 0 \\
\hline
\end{tabular}

\footnotetext{
a Subscript "L" designates the Landolt (1983) version of $(V-R)_{\mathrm{C}}($ see $\S 6$ of Taylor \& Joner 1996).

b Units are mmag arcminute ${ }^{-1}$. If a quantity is zero by assumption, no standard error is quoted.

c Units are mmag. If a quantity is zero by assumption, no standard error is quoted.

${ }^{\mathrm{d}}$ Because $C_{\alpha} \neq 0$ at the $4 \sigma$ level, results of $I_{\mathrm{C}}$ and $(V-I)_{\mathrm{C}}$ positional tests of extrinsic data given by Laugalys et al. are deemed to be superseded.

e False-discovery rate (see Miller et al. 2001) shows that neither $C_{\alpha}$ nor $C_{\delta}$ differs from zero at an overall confidence rate of 0.95 .
}

through 1992. The telescopes used include the $1.3 \mathrm{~m}$ and Number 2 $0.9 \mathrm{~m}$ telescopes at Kitt Peak National Observatory (KPNO). Measurements were also made at the $1 \mathrm{~m}$ telescope of Cerro Tololo Inter-American Observatory (CTIO) and the $0.6 \mathrm{~m}$ telescope of the West Mountain Observatory of Brigham Young University. The observing techniques used at these telescopes and the reduction procedures applied to the data have been described in $\S 2.1$ of Joner \& Taylor (1990). Some (although not all) of those data have been published in Joner \& Taylor $(1988,1990)$ and Taylor \& Joner $(1985,1988)$.

A second database is taken from M67 frames taken in 1993 December and 1995 January at the $0.9 \mathrm{~m}$ telescope of CTIO. The detector used at that telescope was a Tek1024 Number 2 CCD. The third database is taken from M67 frames obtained in 1992 February at the $0.6 \mathrm{~m}$ Burrell-Schmidt telescope at KPNO. At that telescope, the detector was an S2KA CCD mounted at the Newtonian focus. In this case, only data from a subframe of $601 \times 601$ pixels were used.

At each telescope where CCD photometry was performed, only data from nights deemed to be photometric were retained. We stress the fact that both the photomultiplier and CCD data are solely from measurements made on such nights. When CCD cameras were used, an average of 20 bias frames was obtained on each night. Flat frames were taken through each of the adopted filters at twilight. Both cameras were cooled using liquid nitrogen, so no dark frames were required in the reductions. After initial processing was done using the bias and flat frames, aperture photometry was performed to extract raw magnitudes. Intermediate processing then yielded instrumental values of $V,(V-R)_{\mathrm{C}}$, and $(R-I)_{\mathrm{C}}$. Those data were reduced with the BIGPHOT program, which was also used to reduce the photomultiplier data (see $\S 2.1$ of JT90).

The standard stars used in this program are in M67 or are listed by Landolt (1992). However, no standard star data were adopted from the latter source. As in TJ05, all of our adopted standard star data are from photomultiplier measurements reduced to the system of Landolt (1983). For M67 stars in particular, the standardstar data were taken from TJ85 and JT90.

After initial reductions of the CCD data were complete, the data were tested in two ways. In both sets of tests, the CCD data and the photomultiplier data were differenced. The first test consisted of a search of the resulting residuals for gradients across the face of the cluster. These tests were prompted by an illuminating discussion by Laugalys et al. (2004), who used photomultiplier data to deduce that there are gradient errors in a number of published CCD data sets for M67. Because no flat-fielding procedure is required in photomultiplier photometry, the assumption that such photometry is less likely to suffer from gradient errors than CCD data seemed plausible to us. We therefore applied the same basic procedure that Laugalys et al. did. However, we used least-squares analysis in place of the graphical technique used by those authors. The adopted regression equation has the form

$$
\Delta(\text { Index })=C_{\alpha} \Delta \alpha+C_{\delta} \Delta \delta+C_{0}
$$

with "Index" designating $V,(V-R)_{\mathrm{C}}$, and $(R-I)_{\mathrm{C}}$ in turn. The quantity $\Delta \alpha$ is an offset in right ascension, while $\Delta \delta$ is the corresponding declination offset. In both cases, the offsets are from the center of M67 given in SIMBAD.

To decide whether gradient errors had been found, it was necessary to test the regression coefficients $\left(C_{\alpha}, C_{\delta}\right.$, and $\left.C_{0}\right)$ for statistical significance. This was done by using $t$-tests and falsediscovery rate (see $\S 4.2$ of Taylor \& Joner 2006 and $\S 3$ of Miller et al. 2001, respectively). In two cases, it was found that at least one of the derived coefficients is significant at an overall confidence level of $95 \%$ or better. These coefficients (see the first two lines of Table 1) were then used to make the required corrections.

When these tests and corrections were complete, the zero points of the photomultiplier and CCD data were compared. If statistically significant offsets were found, the CCD data were corrected to the zero points of the photomultiplier data. For the CTIO results, the mean remaining offsets from the photomultiplier zero points are

$\left[\Delta V, \Delta(V-R)_{\mathrm{C}}, \Delta(R-I)_{\mathrm{C}}\right]=[3 \pm 2,-1 \pm 1,1 \pm 1] \mathrm{mmag}$.

For the KPNO data, the corresponding equation is

$\left[\Delta V, \Delta(V-R)_{\mathrm{C}}, \Delta(R-I)_{\mathrm{C}}\right]=[3 \pm 2,0 \pm 2,-2 \pm 1]$ mmag.

As one can see from inspection, none of these offsets have absolute values that exceed twice their standard errors, so none of the offsets are statistically significant.

\section{ASSESSING M67 COLOR INDICES: FIRST STEPS TOWARD A SOLUTION}

With the augmented M67 database in hand, we consider the M67 zero-point problem described in $\S 1$. A number of M67 VRI data sets have been published (see $\S 5$ ), but not all of them have 
played an active role in the problem. The databases that have been featured fall naturally into a "blue group" and a "red group." The blue group contains the JT90 data and a data set from Sandquist (2004), while the red group contains data sets from Montgomery et al. (1993) and Richer et al. (1998). According to VandenBerg \& Stetson (2004, hereafter VdBS) the zero-point separation between these groups is about $0.02 \mathrm{mag}$. Those authors conclude that the zero point of the red group is probably correct.

To approach this problem, we begin by adopting two protocols. One is the so-called FM (for "few millimag") standard of data precision and accuracy (see $\S 2$ of T06). According to this standard, photometric quantities can be meaningful if they range down to a few millimags. For averages specifically, errors as small as about $1 \mathrm{mmag}$ are deemed to be acceptable. Readers who are unfamiliar with the FM standard are invited to consult $\S 7.3$ of TJ06 and $\S 11$ of this paper.

The second adopted protocol is statistical analysis. Before now, it appears that statistical procedures have not been applied to the zero-point problem. The procedure that has been used most often has been graphical fitting of isochrones to plotted color magnitude data (see, e.g., Figs. 4-6 of VdBS). Judging by statistical standards, the salient weakness of that procedure is its inability to yield rigorous confidence limits for deduced quantities (see $\S 4.1$ of Taylor 2001a). We therefore conclude that graphical color magnitude inference is not genuinely trustworthy and suggest that it be displaced by statistical analysis in the future.

The statistical procedures adopted here include two least-squares algorithms (see $\S 2.1$ of TJ06). Their output residuals are tested for wild points by using the Thompson $t$-test (see the second tool described in $\S 6.2$ of Taylor 2000). The statistical significance of the coefficients they yield is evaluated by using ordinary $t$-tests. In addition, an algorithm for analyzing differences between data vectors is applied. Each application of the algorithm yields a mean difference between the vectors and an estimated rms error for the data in one of them. A detailed derivation of this algorithm is given in Part 2 of Appendix C of Taylor (1991). However, interested readers should probably consult a summary description of the algorithm instead (see Appendix C of JT90 or $\S 6.2$ of T00).

\section{ASSESSING M67 COLOR INDICES: DETAILED PROCEDURE}

\subsection{Analysis Tactics}

Our next step is to select a specific way of performing the analysis. To begin, we set aside two procedures used by VdBS. Those authors use plots of $B-V$ against $(V-I)_{\mathrm{C}}$ for cluster and field stars. The problem with this technique is that the use of $B-V$ leads to ambiguities that have nothing to do with the accuracy of the $(V-I)_{\mathrm{C}}$ data that are being tested. This is true partly because $B-V$ data sets can have their own zero-point offsets and partly because $B-V$ is sensitive to blanketing. As a result, $B-V$ is sensitive to both metallicity and to inherent scatter in the relation between metallicity and blanketing (for a brief discussion of such scatter, see $\S 5.3$ of T06).

$\mathrm{VdBS}$ also gauge data accuracy by comparing $\left[M_{V},(V-I)_{\mathrm{C}}\right]$ main-sequence loci for M67 and NGC 188 (see especially their Fig. 5). To do this, they must obviously adopt data for NGC 188 , and they also require reddening values for both that cluster and M67. At the moment, their adopted reddening value for M67 is supported by a reasonably comprehensive analysis (see $\S 9$ of $\mathrm{T} 07 \mathrm{a}$ ), but their reddening value for NGC 188 is not. In this case, ambiguity is therefore introduced by their choice of a reddening value for NGC 188 and also their choices of photometry for that cluster. One notes that Stetson et al. (2004), who use the same approach as VdBS, concede (at least pro forma) that their deduced results could be influenced by a "pernicious conspiracy" among systematic errors in contributing quantities. All told, eliminating any possibility for such a conspiracy would be worthwhile.

The alternative approach we adopt consists of a series of comparisons between data vectors. When $(V-R)_{\mathrm{C}}$ is analyzed, for instance, the vectors contain values of $(V-R)_{\mathrm{C}}$ that are drawn from diverse sources, but apply for the same selection of stars. Equivalent procedures are used for $(R-I)_{\mathrm{C}}$ and $(V-I)_{\mathrm{C}}$. We stress the fact that in this procedure, the only participating quantity besides the data sets being tested is the M67 reddening (see $\S 4.4$ ). As a result, ambiguities like those noted above are minimized.

In most cases, the results derived from the two vectors are formal zero-point differences. For M67 data, however, some tests for differing scale factors are performed as well. In these cases, linear regression relations between vectors are calculated. The result of each test is then stated as a value of

$$
s \equiv 100(S-1)
$$

where $S$ is the slope of the calculated relation. If $s \neq 0$ at $95 \%$ confidence or better, it is concluded that a scale factor difference between the vectors has been found.

\subsection{Groups of Comparisons}

Three groups of comparisons between the augmented M67 database and other data sets are performed. One group is inspired by the choice of standard star data by Montgomery et al. (1993). Those authors note that some of those data are from JT90. If the JT90 data are in error while those of Montgomery et al. are not, the Montgomery et al. reductions must have yielded a zero-point error that largely or entirely compensates for the one affecting the JT90 data. VdBS do not note this point, so they do not acknowledge that such a coincidence seems unlikely prima facie. A pertinent way to gauge this possibility is to find out whether other observers who have used the JT90 standards (either directly or indirectly) have derived results on the Montgomery et al. zero point. Tests are performed to see whether this has in fact happened.

A second group of comparisons focuses on data based on sets of standard stars that are completely disjointed from the set we have used. In this case, both zero-point and scale factor comparisons are performed. Note that if agreement is found in these cases, it cannot be dismissed as a simple artifact of the use of common standard stars. In addition, tests of this sort can now include comparisons between the augmented M67 database and a data set based directly on the $\mathrm{E}$ region standards (for a collection of $\mathrm{E}$ region standard star data, see, e.g., Menzies et al. 1989). For both reasons, this set of comparisons is deemed to be important.

A third group of comparisons is a response to the problem of reducing photometry to a standard system. It has been known for some time that if measurements of two or more groups of stars are transformed independently to a standard system, zero-point differences can result (see, e.g., $§ 2$ of Strom et al. 1971 and Table 3 of Stetson et al. 2004). Such differences are much less likely, however, if photometric nights are used to perform direct comparisons of the groups at the telescope. Sturch $(1972,1973)$ appears to have been the first to publish fully documented exercises of this sort. We follow Sturch's procedure here and refer to it with the phrases "Sturch comparison" and "Sturch exercise."

\subsection{Sturch Comparisons: A Two-Step Process}

We use Sturch comparisons as part of a two-step exercise in which an indirect link is established between M67 and the E region standards. This is done by treating the Hyades as a northern 
TABLE 2

REDDENING RATIOS

\begin{tabular}{|c|c|c|}
\hline Ratio & Value & Source \\
\hline$A_{V} / E(B-V) \ldots \ldots \ldots \ldots \ldots \ldots$ & 3.28 & Buser (1978), Table $6^{\text {a }}$ \\
\hline$A_{V} / E(b-y) \ldots \ldots \ldots \ldots \ldots \ldots$ & 4.27 & Crawford \& Mandwewala (1976), Table $\mathrm{X}^{\mathrm{a}}$ \\
\hline$E(b-y) / E(B-V) \ldots \ldots \ldots$ & 0.77 & $\ldots{ }^{b}$ \\
\hline$E(V-R)_{\mathrm{C}} / E(B-V) \ldots .$. & 0.58 & Taylor (1986), Table $3^{\mathrm{c}}$ \\
\hline$E(R-I)_{\mathrm{C}} / E(B-V) \ldots \ldots$ & 0.70 & Taylor (1986), Table $3^{\mathrm{c}}$ \\
\hline$E(V-K) / E(B-V) \ldots \ldots$ & 2.63 & Cardelli et al. (1989) ${ }^{\mathrm{d}}$ \\
\hline
\end{tabular}

a The quoted ratio is based on the Whitford (1958) reddening law and applies at spectral type F0.

b The quoted ratio follows from the two entries just above.

${ }^{c}$ See Table 2 of this source for a review of ratios from diverse reddening laws. et al.

d The quoted ratio is based on an averaged reddening law derived by Cardelli

hemisphere proxy for those standards. Extensive Cousins VRI photometry of the Hyades has been published, so a review of the scale factor and zero-point status of those data is the first of the two steps.

In the second step, Hyades measurements are used to establish zero points for M67 data. The data sets adopted for the indirect comparison include data for both clusters. Some of those data are from Sturch comparisons, while the remainder are from data sources that appear likely to have uniform zero points. The M67 data in the adopted data sets are more extensive than those available for the direct comparison to the $\mathrm{E}$ region standards. In addition, the indirect comparison is based partly on measurements of stars on and near the M67 main sequence. This condition does not hold for the direct comparison, which is limited to data for giants and blue stragglers in M67 because those data have been secured with a photomultiplier and a $0.5 \mathrm{~m}$ telescope. For these reasons, the direct and indirect comparisons yield complementary links to the E region standards.

\subsection{Use of Transformations}

The data used in the Sturch comparisons are not on the Cousins system, although they are on comparable systems. To deal with this problem, color-color transformations are applied. Those transformations have been derived rigorously by using procedures given in a tutorial by TJ06. In addition, they are based partially or wholly on Hyades data, as required by the procedure adopted here. Allowances for the errors introduced by the transformations are made by using a discussion in $\S 7.1$ of TJ06.

We acknowledge that to a number of readers, the use of transformations is likely to appear to be self-defeating. Skepticism about the accuracy of transformed data has been fairly common for some time (for a recent example, see $\S 2$ of Ramírez \& Meléndez 2005). A concise response to this issue is given below (see $\S 11$ ). Readers with fundamental questions about transformations are invited to consult TJ06 as well. That paper contains an extended discussion of the derivation and use of accurate transformations.

For some data, the original wavelength baselines are quite different from those of the Cousins system. In those cases, the transformations have some reddening dependence. The reddening ratios required here are given in Table 2 together with their sources. The required values of $E(B-V)$ are adopted from a series of detailed analyses by T06, T07a, and T07b. Readers with questions about the accuracy of the adopted reddening values are invited to consult those papers. To assess the effects of reddening uncertainties on the data comparisons, we note that the largest
TABLE 3

Hyades and Landolt (1983) Data: Scale and Zero-Point Tests

\begin{tabular}{|c|c|c|c|c|}
\hline Source of Tested Data & Index & $s^{\mathrm{a}}$ & $\begin{array}{l}\text { Offset }^{\mathrm{b}} \\
(\mathrm{mmag})\end{array}$ & $\begin{array}{l}\text { Reddest Color } \\
\text { (mag) }\end{array}$ \\
\hline Landolt $(1983)^{\mathrm{c}}$ & $(V-R)_{\mathrm{C}}$ & d & $0 \pm 3$ & 0.80 \\
\hline Landolt $(1983)^{\mathrm{e}}$. & $(R-I)_{\mathrm{C}}$ & $0.1 \pm 0.2$ & $0.1 \pm 0.5$ & 1.45 \\
\hline Hyades $(\mathrm{TJ} 85)^{\mathrm{f}}$. & $(V-R)_{\mathrm{C}}$ & $0.0 \pm 1.4$ & $-3.5 \pm 1.3^{\mathrm{g}}$ & 0.51 \\
\hline Hyades $(\mathrm{TJ} 85)^{\mathrm{f}} .$. & $(R-I)_{\mathrm{C}}$ & $-2.1 \pm 1.0^{\mathrm{g}}$ & $-0.9 \pm 1.0$ & 0.51 \\
\hline Hyades (TJ05) ${ }^{\mathrm{f}}$. & $(R-I)_{\mathrm{C}}$ & $-0.9 \pm 0.8$ & $0.1 \pm 1.0$ & 0.51 \\
\hline
\end{tabular}

a $s \equiv 100(S-1)$.

b This quantity is the formal correction to be added to the data being tested.

c The quoted results are from $\S 6$ of TJ96. The confidence interval is not a $\pm 2 \sigma$ interval but instead includes the maximum corrections required if eq. (5) of TJ96 (which is a compromise relation) is adopted.

d The scale factor difference obtained here appears in eq. (5).

e The quoted results are from Table 3 of TJ96.

${ }^{\mathrm{f}}$ The quoted results are from Table 3 of Joner et al. (2006).

g Although $t>2$ for this datum, it does not differ from zero at $95 \%$ overall confidence when tested using false-discovery rate (see Miller et al. 2001).

quoted standard error for an adopted value of $E(B-V)$ is 4 mmag. Numerical tests show that in the worst case considered [conversion of $V-K_{2}$ to $(R-I)_{\mathrm{C}}$ ], the effect of an error of that size is scaled down by a factor of 5 . In the best case [conversion of $(R-I)_{\mathrm{J}}$ to $(R-I)_{\mathrm{C}}$ ], the induced error is even closer to 0 . We therefore conclude that even in the context of the FM standard, the effects of reddening uncertainties can be neglected.

\section{ASSESSING M67 COLOR INDICES: FIRST TESTS AND RESULTS}

\subsection{Testing Hyades Data}

To apply the procedure just described, we begin by reviewing the status of Cousins VRI Hyades photometry given by TJ85 and TJ05. Since all of that photometry is on the standard system of Landolt (1983) the status of Landolt's data is reviewed as well. To test the Landolt data, extrinsic results from sources given by Taylor \& Joner (1996) are used. To test the Hyades data, extrinsic results given by Joner et al. (2006) are used. ${ }^{3}$ All tests refer the tested data to the E region standards, and all are made using measurements for more than 35 stars. In addition, all data used in the tests have rms errors ranging from 2 to $6 \mathrm{mmag}$.

For $(V-R)_{\mathrm{C}}$, a scale factor difference between the Landolt and $\mathrm{E}$ region data is found:

$$
(V-R)_{\mathrm{L}}=1.011(V-R)_{\mathrm{C}},(V-R)_{\mathrm{C}}<0.8 \mathrm{mag},
$$

with the subscript "L" referring to the Landolt system (see eq. [5] of TJ96). As a result, all other $V-R$ tests refer the tested data to a fictitious database produced by applying equation (5) to $E$ region values of $(V-R)_{\mathrm{C}}$. In Table 3 , results from both comparisons of this kind and $(R-I)_{\mathrm{C}}$ comparisons are given. The first two entries in the table show that if data for M stars are excluded (as they are throughout this paper), it has been possible to secure accurate Hyades data by using the Landolt (1983) standard stars. The last three entries show that there are no detectable differences between the Hyades data of TJ85 and TJ05 and Hyades data standardized by using E region standards. We conclude that combined data from all those sources are on the $\mathrm{E}$ region system at the level required by the FM standard.

\footnotetext{
${ }^{3}$ For clarity of reference, we say that "extrinsic" data are used to establish the status of "tested" data.
} 


\subsection{Assembling M67 Data}

To establish the link from the Hyades to M67, the databases listed in Table 4 are used. The databases labeled $R_{1}, I_{1}$, and $I_{2}$ in the table are from Sturch comparisons. For database $I_{3}$, that condition does not hold. However, the data in that database are from an all-sky survey that appears likely to have a uniform zero point (see Cutri et al. 2003). ( The footnotes to Table 4 give further information, including references to Appendix A for discussions of some required color-color transformations.)

To identify other extrinsic databases that can be considered here, the WEBDA database has been consulted. Extrinsic Cousins data published before 1990 are not used here because they have been discussed by JT90. Measurements made by Stassun et al. (2002) are excluded because they have not been fully reduced to a standard system. All other published Cousins VRI data sets listed by WEBDA are included along with data from Laugalys et al. (2004). Extrinsic data based directly from E region standards are from a forthcoming paper (see M. D. Joner et al. 2008, in preparation).

\subsection{Testing M67 Data}

The results of the M67 data tests are given in Table 5. In this case, the tested data have rms errors ranging from 3 to $8 \mathrm{mmag}$, and this range also holds for extrinsic data for which no rms errors are listed. The fourth through sixth columns of the table contain numbers of data pairs, calculated formal offsets, and derived rms errors for extrinsic data, respectively. By comparing entries in those three columns, one can see how the standard errors listed for the offsets follow from the number and precision of the contributing data.

For the first five data sources listed in Table 5, the label " $\mathrm{M}$ " is used. These are the tests designed to see whether authors who have used the JT90 standards have recovered results like those of Montgomery et al. (1993; see our $\S 4.2$ ). For one of the five labeled tests, the result must be set aside because a positional gradient is detected (see the entry for Laugalys et al. 2004). Note, however, that three other tests do not recover the Montgomery et al. offset (which is given in the uppermost boldface entry in the "Difference" column). Statistical testing underscores this conclusion: the offset for the Montgomery et al. data differs from zero at a very high confidence level $(P>4.7)$, while $P<0$ for the other three entries. ${ }^{4}$ Even before the remaining results are reviewed, one must suspect that the Montgomery et al. data suffer from a zero-point error that they alone possess.

Just after the Table 5 entries for Montgomery et al. data are entries for Sandquist (2004) data. The latter are given out of sequence so that they may be compared at once to their counterparts for Montgomery et al. Note that the rms error derived for the Sandquist data is quite a bit smaller than its counterpart for the Montgomery et al. data (look at the second line with boldface entries in Table 5). In addition, the Sandquist data have an offset that is smaller in absolute value. The implications of this second result will be considered shortly.

The next three offsets listed in Table 5 describe test results for $(V-R)_{\mathrm{L}}$. None of them differ from zero with $P>0$. From the Mendoza (1967) data, one finds that the $2 \sigma$ limit of the formal

\footnotetext{
4 If the confidence level $C$ is very close to unity, it must be expressed with a row of nines that can make the number hard to grasp. A more accessible alternative is to let $p \equiv 1-C$ be the probability of Type I error for an isolated test and then define $P$ as $-\log _{10}(20 p)$, as is done here. It is useful to remember that $P=0$ if $C=0.95$ and $P=1$ if $C=0.995$, with the latter value leading to decisive rejection of a null hypothesis in almost all cases.
}

TABLE 4

Hyades, Coma, M67: Data Sets for Zero-Point Tests

\begin{tabular}{|c|c|c|c|c|c|}
\hline Data Set & Index & Hyades & Coma & M67 & Sources \\
\hline$R_{1} \ldots \ldots \ldots \ldots \ldots$ & $(V-R)_{\mathrm{J}}$ & $\mathrm{Y}$ & . & $\mathrm{Y}$ & Mendoza $(1967)^{a, b}$ \\
\hline$I_{1} \ldots \ldots \ldots \ldots \ldots \ldots$ & $b-y$ & $\mathrm{Y}$ & $\mathrm{Y}$ & $\mathrm{Y}$ & TJ92, JT97 ${ }^{\text {c }}$ \\
\hline$I_{2} \ldots \ldots \ldots \ldots \ldots \ldots$ & $(R-I)_{\mathrm{J}}$ & $\mathrm{Y}$ & $\cdots$ & $\mathrm{Y}$ & Mendoza (1967) ${ }^{\mathrm{a}, \mathrm{d}}$ \\
\hline$I_{3} \ldots \ldots \ldots \ldots \ldots \ldots$ & $V-K_{2}$ & $\mathrm{Y}$ & $\mathrm{Y}$ & $\mathrm{Y}$ & Cutri et al. $(2003)^{\mathrm{e}}$ \\
\hline
\end{tabular}

${ }^{a}$ V. E. E. Mendoza (1977, private communication) affirms the zero-point uniformity of these data. See $\S$ IV of TJ85 for the reason Coma data from this source are omitted.

${ }^{b}$ See item 1 of Appendix A for a review of transformations for these data.

${ }^{c}$ For a transformation from $b-y$ to $(R-I)_{\mathrm{C}}$, see Table 4 of TJ06.

d For the required transformations from $(R-I)_{\mathrm{J}}$ to $(R-I)_{\mathrm{C}}$, see eqs. (A1)(A5c) in Appendix A of TJ05. These transformations allow for a problem with the Mendoza data discussed in $\S 4.3$ of TJ05.

e For a review of transformations for these data, see item 2 of Appendix A.

zero-point adjustment is $10.8 \mathrm{mmag}$. This means that the data do not rule out a zero-point adjustment of that size with $P>0$. In the context of the FM standard, this is a relatively large uncertainty. Fortunately, the entries based on the Gilliland et al. and SAAO data yield $2 \sigma$ limits of 3.6 and $3.2 \mathrm{mmag}$, respectively. Those limits are quite satisfactory.

The last five entries in Table 5 apply for $(R-I)_{\mathrm{C}}$. In this case, it appears at first that a result comparable to the one for $(V-R)_{\mathrm{L}}$ will be obtained at once. For the first four entries, $P$ is found to be $<0$. Here again, the Mendoza (1967) data yield a fairly large $2 \sigma$ limit (9.4 mmag). However, the other two Sturch comparisons suggest that that limit may be as small as $1.6 \mathrm{mmag}$ (see the entry for data set $I_{3}$ ). A separate test for giants yields consistency with a consensus of independent data (see the second entry from the bottom of Table 5). However, the entry for the SAAO data yields an offset of $-6 \pm 1.6 \mathrm{mmag}$ (see the boldface entry on the last line of the table). In this case, the value of $P$ is 1.33 .

Fortunately, this result does not imply that one must make a choice between the zero points implied by the direct and indirect methods. Instead, the Table 5 offsets are reassessed after a 2 mmag correction has been subtracted from the $(R-I)_{\mathrm{C}}$ data in the augmented M67 database. It is assumed that if the JT90 data had included this correction, M67 data based on JT90 standards would also have included it, leaving the resulting formal zero-point differences unaltered. For this reason, Table 5 entries for data sets flagged with an "M" are not adjusted. For entries flagged with a double asterisk, however, 2 mmag are added to the quoted offsets. The entire list of revised offsets (which does not appear in Table 5) is then retested by using false-discovery rate (Miller et al. 2001) and Student's $t$-tests.

When this procedure is carried out, it is found that the revised $(R-I)_{\mathrm{C}}$ zero point does not differ from either the direct or indirect zero points with $P>0$. The only offsets that continue to be statistically significant are those for the Sandquist and Montgomery et al. data. Note that since the 2 mmag adjustment is made in response to statistical testing and yields superior results from such testing, it cannot be fairly regarded as an ad hoc device.

\section{ASSESSING M67 COLOR INDICES: FURTHER RESULTS FOR TWO IMPORTANT PAPERS}

The test results given above for the Montgomery et al. and Sandquist data sets warrant further development. We therefore compare the zero points of those data sets to the zero points of the E region standards. Fortunately, the augmented M67 database can be used as proxies for those standards if one allows for 
TABLE 5

M67 VRI Colors: Offsets and Accidental Errors

\begin{tabular}{|c|c|c|c|c|c|}
\hline Extrinsic Source & Extrinsic Color ${ }^{\mathrm{a}}$ & Tested Color ${ }^{a}$ & $n^{\mathrm{b}}$ & $\begin{array}{l}\text { Difference }^{\mathrm{c}} \\
(\mathrm{mmag})\end{array}$ & $\begin{array}{c}\sigma^{\mathrm{d}} \\
(\mathrm{mmag})\end{array}$ \\
\hline M: Anupama et al. (1994) ${ }^{\mathrm{e}}$.. & $(V-I)_{\mathrm{C}}$ & $(V-I)_{\mathrm{C}}$ & 30 & $2 \pm 4.7$ & 24 \\
\hline M: Chevalier \& Ilovaisky $(1991)^{\mathrm{e}}$. & $(V-R)_{\mathrm{L}}$ & $(V-R)_{\mathrm{L}}$ & 29 & $2 \pm 1.5$ & 7 \\
\hline M: Chevalier \& Ilovaisky $(1991)^{\mathrm{e}, \mathrm{f}} \ldots \ldots$. & $(R-I)_{\mathrm{C}}$ & $(R-I)_{\mathrm{C}}$ & 29 & $6 \pm 2.7$ & 14 \\
\hline M: Laugalys et al. (2004) ${ }^{\mathrm{e}, \mathrm{g}}$.. & $(V-I)_{\mathrm{C}}$ & $(V-I)_{\mathrm{C}}$ & 202 & & 10 \\
\hline M: Montgomery et al. $(1993)^{\mathrm{e}}$.. & $(V-I)_{\mathrm{C}}$ & $(V-I)_{\mathrm{C}}$ & 172 & $25 \pm 1.3$ & 16 \\
\hline${ }^{* *}$ Sandquist (2004) & $(V-I)_{\mathrm{C}}$ & $(V-I)_{\mathrm{C}}$ & 78 & $-6 \pm 1.0$ & 6 \\
\hline$R_{1}$ : Mendoza $(1967)^{\mathrm{h}}$ & $(V-R)_{\mathrm{J}}$ & $(V-R)_{\mathrm{L}}$ & 37 & $6 \pm 5.4$ & 33 \\
\hline Gilliland et al. $(1991)^{\mathrm{f}}$ & $(V-R)_{\mathrm{L}}$ & $(V-R)_{\mathrm{L}}$ & 63 & $-4 \pm 1.8$ & 13 \\
\hline SAAO $^{\mathrm{i}}$ & $(V-R)_{\mathrm{L}}$ & $(V-R)_{\mathrm{L}}$ & 11 & $0 \pm 1.6$ & $\ldots$ \\
\hline . & $b-y$ & $(R-I)_{\mathrm{C}}$ & 35 & $-2 \pm 1.5$ & $\ldots$ \\
\hline${ }^{* *} I_{2}$ : Mendoza $(1967)^{\mathrm{h}}$. & $(R-I)_{\mathrm{J}}$ & $(R-I)_{\mathrm{C}}$ & 39 & $-6 \pm 4.7$ & 15 \\
\hline${ }^{* *} I_{3}$ : Cutri et al. $(2003)^{\mathrm{k}} \ldots$. & $V-K_{2}$ & $(R-I)_{\mathrm{C}}$ & 242 & $0 \pm 0.8$ & $\ldots$ \\
\hline 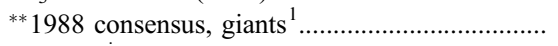 & $\ldots$ & $(R-I)_{\mathrm{C}}$ & 12 & $-2 \pm 1.3$ & $\ldots$ \\
\hline${ }^{* *} \mathrm{SAAO}^{\mathrm{i}, \mathrm{m}}$ & $(R-I)_{\mathrm{C}}$ & $(R-I)_{\mathrm{C}}$ & 11 & $-6 \pm 1.6$ & $\ldots$ \\
\hline
\end{tabular}

${ }^{\text {a }}$ Subscript "L" designates the Landolt (1983) version of $(V-R)_{\mathrm{C}}$ (see $\S 6$ of TJ96).

b The quantity $n$ is the number of stars for which data are compared.

${ }^{\mathrm{c}}$ Differences are in the sense "extrinsic source minus this paper" and are expressed as values of the tested color index.

${ }^{\mathrm{d}}$ The quantity $\sigma$ is the rms error per data entry for data from the extrinsic source. No value of $\sigma$ is given if accidental errors for the extrinsic data are already known independently.

e Data from this source have been derived (directly or indirectly) using standard-star data from JT90.

${ }^{\mathrm{f}}$ The zero point of the data used to standardize the Gilliland et al. results is known to be consistent with the zero point of the augmented M67 database (see $\S 5$ of JT90).

${ }_{\mathrm{g}}$ No difference is given here because a positional correction is required instead (see Table 1).

${ }^{\text {h }}$ Data for giants are used in the $(V-R)_{\mathrm{L}}$ test, but not in the $(R-I)_{\mathrm{C}}$ test.

i The extrinsic data used here are given by M. D. Joner et al. (2008, in preparation).

${ }^{j}$ Applied luminosity corrections are from Fig. 6 of Crawford \& Barnes (1970) and the $\delta c_{1}$ term in the Crawford (1975) calibration. Data for giants are excluded from the analysis

${ }^{\mathrm{k}}$ The $V$ data required for this index are taken from this paper. Data for giants are excluded from the analysis.

1 The extrinsic data for this test are averaged from the following sources: $(R-I)_{\mathrm{K}}$ (Brooke 1969), $T_{1}-T_{2}$ (Canterna 1976), CTIO/ CIT $V-K$ (Cohen et al. 1978), and $(R-I)_{\mathrm{J}}$ (Mendoza 1967). For further information, see Table 1 of TJ88.

$\mathrm{m}$ The quoted offset is statistically significant before (but not after) the zero-point correction described in the text is applied.

the uncertainties in the zero-point relationship between the augmented M67 data and the E region data. The zero-point differences found in this way are as follows:

1. $\Delta(V-I)_{\mathrm{C}}=27 \pm 2.9$ mmag ("Montgomery et al. minus E region");

2. $\Delta(V-I)_{\mathrm{C}}=-4 \pm 2.7$ mmag ("Sandquist minus $\mathrm{E}$ region").

Note that the zero point of the Montgomery et al. data differs decisively from that of the $E$ region standards, while the $E$ region and Sandquist zero points are indistinguishable. (The calculation required to obtain these results is given in Appendix B.)

One would like to know whether scale factor differences can also be found. If the Gilliland et al. and Sandquist data are each compared to the augmented M67 data in turn, one finds that $s=0.4 \pm 0.7$ and $s=2.1 \pm 1.4$, respectively. (Recall that the method used to obtain these results and the definition of $s$ are described in $\S 4$.) Evidently, the augmented database shares a common $(V-R)_{\mathrm{L}}$ scale factor with the Gilliland et al. data and a common $(V-I)_{\mathrm{C}}$ scale factor with the Sandquist data. On the other hand, if the augmented database and the Montgomery et al. data are compared, one finds that $s=2.7 \pm 1.1$ and $P=0.55$. In addition, Sandquist's Figure 5 suggests that there is a corresponding scale factor difference between his data and those of Montgomery et al. Prima facie, it therefore appears that the zeropoint offset of the Montgomery et al. data is accompanied by a scale factor offset. Although that assessment should be checked by using additional databases, it is accepted here on an interim basis.

These results have a consequence worth noting. An et al. (2007) have derived M67 distance moduli from color-magnitude analyses of both the Montgomery et al. and Sandquist data. Pending an updated analysis, their distance modulus based on the Sandquist data should be used (see their Table 7).

\section{SOME COMMENTS ABOUT THE VdBS ANALYSIS}

Recall at this point that the VdBS analysis favors the zero point of the Montgomery et al. data. Although general questions have been raised about the VdBS analysis techniques (recall $\S 4.1$ ), obviously it would ultimately be best to rely on pertinent specifics. If this is done, does one find that the results of the two analyses are implacably opposed to each other? Fortunately, we can show that this is not the case.

The issue of interest here is a problem first discussed by VandenBerg \& Clem (2003, see $\S \S 3.2$ and 3.6 of their paper). For stars with solar metallicity, those authors use the data of Montgomery et al. to calibrate their $(V-I)_{\mathrm{C}}$ isochrones. Subsequently, they fit isochrones to color-magnitude diagrams for the Hyades (see their Figs. 22-24). For $(V-I)_{\mathrm{C}}$, they find that the plotted data are about 0.02 mag bluer than the isochrone, with the offset being almost independent of color. In contrast, no comparable problem appears for $B-V$ or $V-R$ (compare Fig. 24 of VandenBerg \& Clem to their Figs. 22 and 23). ${ }^{5}$

One way to explain this problem is to attribute it to the $(V-$ $I)_{\mathrm{C}}$ data of Montgomery et al. Both VandenBerg \& Clem and VdBS consider this hypothesis, although they do not ultimately accept it. Now that we have shown that it is very likely to be

${ }^{5}$ For the sake of clarity, it should be noted that the designation $(V-R)_{0}$ used by VandenBerg \& Clem corresponds to $(V-R)_{\mathrm{L}}$ in the notation of this paper. This identification applies for both M67 and the Hyades. 
TABLE 6

Hyades, NGC 752, Praesepe: Data Sets for Zero-Point Tests

\begin{tabular}{|c|c|c|c|c|c|}
\hline Data Set & Index & Hyades & NGC 752 & Praesepe & Sources \\
\hline$R_{2} \ldots \ldots \ldots \ldots \ldots \ldots \ldots \ldots \ldots \ldots \ldots \ldots$ & $(V-R)_{\mathrm{J}}$ & Y & $\ldots$ & $\mathrm{Y}$ & Mendoza $(1967)^{\mathrm{a}}$ \\
\hline 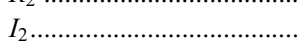 & $b-y$ & $\mathrm{Y}$ & $\mathrm{Y}$ & 1 & TJ92, JT95 ${ }^{\mathrm{b}}$ \\
\hline$I_{3} \ldots \ldots+\cdots$ & $(R-I)_{\mathrm{J}}$ & $\mathrm{Y}$ & $\ldots$ & Y & Mendoza $(1967)^{c}$ \\
\hline$I_{4}$ & $V-K_{2}$ & $\mathrm{Y}$ & Y & $\mathrm{Y}$ & Cutri et al. $(2003)^{\mathrm{d}}$ \\
\hline 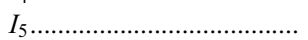 & $V-i *$ & $\ldots$ & $\mathrm{Y}$ & $\ldots$ & Jennens \& Helfer $(1975 a, 1975 b)^{e}$ \\
\hline
\end{tabular}

${ }^{\text {a }}$ See item 1 of Appendix A for a review of transformations for these data.

${ }^{b}$ Praesepe is omitted for a reason given in $\S 9.1$ of T06. For a transformation from $b-y$ to $(R-I)_{\mathrm{C}}$, see Table 4 of TJ06.

${ }^{\mathrm{c}}$ For the required transformations from $(R-I)_{\mathrm{J}}$ to $(R-I)_{\mathrm{C}}$, see eqs. (A1)-(A5c) in Appendix A of TJ05. These transformations allow for a problem with the Mendoza data discussed in $\S 4.3$ of TJ05.

${ }^{\mathrm{d}}$ See item 2 of Appendix A for a review of transformations for these data.

e A transformation for these data is derived in Appendix C.1 of T07b. That transformation links data for NGC 752 to field-star data (see Taylor 1986) instead of Hyades data.

correct, it is worthwhile to explore its consequences. To do this, let the $(V-I)_{\mathrm{C}}$ isochrones of VandenBerg \& Clem be moved 27 mmag to the blue, and let this be done for both the Hyades and M67. Now the M67 isochrone fits the augmented M67 database, while the shift of the Hyades isochrone has at least approximately absorbed the Hyades offset of $0.02 \mathrm{mag}$. As a result, it appears that both isochrones now fit data that we have found to be on the same zero point. Moreover, this appraisal probably does not require revision if the M67 value of $E(B-V)$ adopted by VandenBerg \& Clem is replaced by the reddening value deduced by T07a. By happenstance, the two reddening values differ by only 3 mmag.

For $(V-R)_{\mathrm{L}}$, VandenBerg \& Clem obtain an acceptable fit to the Hyades data of TJ85 (see their Fig. 23). Those data share a zero point with the augmented M67 results and hence with the data of Gilliland et al. (1991; see our Table 5). One therefore expects the M67 isochrone of VandenBerg \& Clem to be an adequate fit to the Gilliland et al. results as well. For stars with $M_{V}<8 \mathrm{mag}$, that turns out to be the case. This test is not very definitive because the plotted Gilliland et al. data are quite scattered (see Fig. 14 in VandenBerg \& Clem). However, it does appear that consistency has again been achieved.

This reasoning is not carried further here because it quickly leads beyond the scope of our paper. However, it is persuasive enough to suggest that the results of a complete analysis using recalibrated VandenBerg \& Clem isochrones might be fully consistent with our data. Acting on a recommendation made in $\S 3$, we suggest that such an analysis be carried out statistically.

\section{ASSESSING COLORS FOR COMA, PRAESEPE, AND NGC 752}

We now return to our own analysis and, with solutions for two program clusters in hand, consider results for the other three. For Coma, some further preparation is required before an analysis can be done. As Table 4 shows, tests of $(R-I)_{\mathrm{C}}$ for Coma can be carried out using data sets $I_{1}$ and $I_{3}$. However, no data sets listed there can be used to test Coma values of $(V-R)_{\mathrm{L}}$. In this case, extrinsic data from measurements described in $\S 3.2$ of TJ05 are adopted.

Further preparation is also required before the new data for Praesepe and NGC 752 are tested. By and large, measurements for those clusters were made on the same nights, so their $(V-R)_{\mathrm{C}}$ and $(R-I)_{\mathrm{C}}$ data may each be tested as a unit. However, those units include only scattered measurements of stars in the other three clusters. To test the new data, a second set of measurements from Sturch comparisons is therefore required. Data sets adopted for this purpose are listed in Table 6 .
Results of zero-point tests for Coma, Praesepe, and NGC 752 are listed in Table 7. This time (in contrast to Table 5), rms errors are not given for extrinsic data sets. Instead, we note that the errors are once again derived or are known in advance and that they tend to be smaller than the errors for M67 because brighter clusters are now being considered. This point should be kept in mind as the formal offsets and numbers of contributing data pairs are inspected (see the last two columns of Table 7).

Note that the first offset listed in Table 7 for Coma applies for $(V-R)_{\mathrm{L}}$, while the other two apply for $(R-I)_{\mathrm{C}}$. One can see at once that all three offsets imply that no adjustment of the Coma zero points is required. Moreover, the $2 \sigma$ uncertainties in the offsets are $\leq 3.0 \mathrm{mmag}$ and so are quite satisfactory.

For Coma, no fully independent data that could be used to perform adequate scale factor tests are known to exist. For this reason, no results from such tests are reported here. Instead, it is assumed that the results of the scale factor tests for M67 apply to Coma as well. That assumption is based on the fact that the Coma data and the photomultiplier data for M67 can be considered as part of a single unit (see, e.g., Table I of TJ85). We recommend that the Coma data be used in further color-magnitude analyses of that cluster to see whether problems posed by previous $V-I$ results recur (see $\S 3.6$ of Pinsonneault et al. 1998).

On the fourth line of Table 7, results are reported from a test of the combined $(V-R)_{\mathrm{L}}$ data for Praesepe and NGC 752. That test appears to be the only one of its kind that is feasible at present. Here again, it is found that a derived offset does not differ from zero with $P>0$. If the Mendoza (1967) data used for the zeropoint test are also used for a scale factor test, the resulting value of $s$ is $4.5 \pm 2.2$. This result is accepted here as evidence of scale factor consistency. However, it should be remembered that the derived standard error for $s$ is relatively large (recall the counterpart errors quoted in $\S 6$ ).

The last two entries for Praesepe and the first two listed for NGC 752 display formal $(R-I)_{\mathrm{C}}$ offsets for those clusters. Data for giants are excluded from all four tests. Here a discrepant result with $P=2.4$ appears (see the upper boldface entry in the last column). Judging from the balance of evidence, however, the common $(R-I)_{\mathrm{C}}$ zero point for the two clusters is correct. If the discrepant result is set aside, the $2 \sigma$ limit on this inference is $3.0 \mathrm{mmag}$ (see the entry for data set $I_{3}$ ).

If the procedure developed above were to be applied here, a value of $s$ applying to $(R-I)_{\mathrm{C}}$ would now be calculated. The procedure adopted instead at this point is to test the color zero point for NGC 752 giants. Since those stars are redder than those cluster stars that are on and near the main sequence, separate zero-point tests for the bluer and redder stars can accomplish 
TABLE 7

Coma, Praesepe, NGC 752: Color Offsets

\begin{tabular}{|c|c|c|c|c|c|}
\hline Cluster & Extrinsic Source & Extrinsic Color ${ }^{\mathrm{a}}$ & Tested Color $^{\mathrm{a}}$ & $n^{\mathrm{b}}$ & $\begin{array}{c}\text { Difference }^{\mathrm{c}} \\
(\mathrm{mmag})\end{array}$ \\
\hline 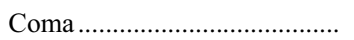 & $\mathrm{TJ} 05^{\mathrm{d}}$ & $(V-R)_{\mathrm{L}}$ & $(V-R)_{\mathrm{L}}$ & 16 & $2 \pm 1.5$ \\
\hline 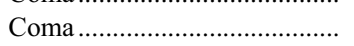 & $I_{1}:$ Crawford \& Barnes (1969), TJ92 ${ }^{\mathrm{e}}$ & $b-y$ & $(R-I)_{\mathrm{C}}$ & 17 & $-1 \pm 1.5$ \\
\hline 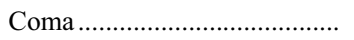 & $I_{3}$ : Cutri et al. $(2003)^{\mathrm{f}}$ & $V-K_{2}$ & $(R-I)_{\mathrm{C}}$ & 18 & $2 \pm 1.4$ \\
\hline 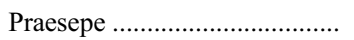 & $R_{1}$ : Mendoza (1967) & $(V-R)_{\mathrm{J}}$ & $(V-R)_{\mathrm{L}}$ & 39 & $3 \pm 2.3$ \\
\hline 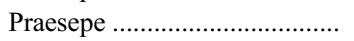 & $I_{2}:$ Mendoza (1967) & $(R-I)_{\mathrm{J}}$ & $(R-I)_{\mathrm{C}}$ & 36 & $3 \pm 3.0$ \\
\hline 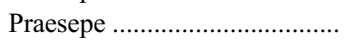 & $I_{3}$ : Cutri et al. $(2003)^{\mathrm{f}}$ & $V-K_{2}$ & $(R-I)_{\mathrm{C}}$ & 38 & $2 \pm 1.5$ \\
\hline 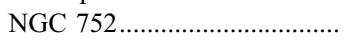 & $I_{1}:$ Crawford \& Barnes (1970), JT95 ${ }^{\mathrm{g}}$ & $b-y$ & $(R-I)_{\mathrm{C}}$ & 19 & $-2 \pm 2.2$ \\
\hline 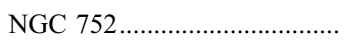 & $I_{3}:$ Cutri et al. $(2003)^{\mathrm{f}}$ & $V-K_{2}$ & $(R-I)_{\mathrm{C}}$ & 29 & $6 \pm 1.4$ \\
\hline$\ldots \ldots \ldots \ldots \ldots \ldots \ldots$ & ${ }^{* *} I_{4}$ : Jennens \& Helfer $(1975 \mathrm{a}, 1975 \mathrm{~b})^{\mathrm{h}}$ & $V-i^{*}$ & $(R-I)_{\mathrm{C}}$ & 7 & $9 \pm 2.7$ \\
\hline NGC $752 \ldots \ldots \ldots \ldots \ldots \ldots \ldots \ldots \ldots \ldots$ & ${ }^{* *} \mathrm{M} 67$ data, this paper ${ }^{\mathrm{i}}$ & $(R-I)_{\mathrm{C}}$ & $(R-I)_{\mathrm{C}}$ & 8 & $-5 \pm 2.9$ \\
\hline
\end{tabular}

Note.-No $b-y$ test is performed for Praesepe because of the color-color offset found for that cluster (see $\S 9$ of T06).

a Subscript "L" designates the Landolt (1983) version of $(V-R)_{\mathrm{C}}$ (see $\S 6$ of TJ96).

${ }^{\mathrm{b}}$ The quantity $n$ is the number of stars for which data are compared.

"Differences are in the sense "extrinsic source minus this paper" and are expressed as values of the tested color index.

" These data have been standardized by using Landolt (1983) standards (see $\S 3.2$ of TJ05).

e The first abbreviation stands for Crawford \& Barnes (1969). The zero-point consistency of data from the two papers is established by TJ92.

${ }^{\mathrm{f}}$ The values of $V$ required for this index are taken from this paper.

g The first abbreviation stands for Crawford \& Barnes (1970). The zero-point consistency of data from the two cited papers is established by JT95.

${ }^{\mathrm{h}}$ This offset is from eq. (C2) of T07b, and applies solely for giants. The adopted value of $E(B-V)$ is from T07b.

${ }^{\mathrm{i}}$ This result is the difference between the formal $(R-I)_{\mathrm{C}}$ corrections derived for M67 and NGC 752 giants by using values of $V-K_{2}$. Allowance is made for the offset listed two entries above this one.

much the same thing as a derivation of $s$. Two tests are performed for the giants (see the entries in Table 7 flagged with double asterisks). Unfortunately, they yield formal zero-point corrections that disagree with $P=1.9$ (compare the lower boldface entry in the last column of the table with the entry just below it). Note, however, that the last result listed in Table 7 is a null correction that is based partly on an M67 zero point that seems to be quite secure (recall $\S 5.3$ ). As a result, the interim judgment adopted here is that no convincing case currently exists for adopting the boldface correction for the giants. The $(R-I)_{\mathrm{C}}$ data for the giants are therefore adopted, although with an acknowledgment that further tests of their zero point should ultimately be made.

\section{9. $V$ MAGNITUDES: ESTABLISHING ZERO POINTS}

Since all of our cluster databases contain $V$ magnitudes as well as colors, zero-point tests for the magnitudes are included. Here the aim of the adopted procedure is to establish all the $V$ magnitudes on the zero point for the Johnson et al. (1966, hereafter JMIW ) measurements of field stars. In one case, cluster data that have been standardized directly to the JMIW system are used (see Table I of Dickens et al. 1968). Otherwise, cluster measurements are linked to that system by using data sets that contain measurements of both cluster stars and JMIW stars. Note that such data sets can be regarded as products of Sturch exercises.

For all the program clusters except M67, the results of the zero-point tests for $V$ magnitudes are given in Table 8 . The first five lines of the table apply for the Hyades and Coma. The first two lines show that for those clusters, there are differences between the adopted zero points and those of Johnson \& Knuckles (1955). The third line shows that the zero point of southern hemisphere Hyades measurements by Joner et al. (2006) is consistent with the zero points of adopted northern hemisphere measurements. ${ }^{6}$ In the

\footnotetext{
${ }^{6}$ Determining the consistency of $V$ zero points for the northern and southern hemispheres has been a concern for some time (see, e.g., $\S 4$ of Taylor \& Joner 1996). For the sake of caution, the result quoted here should probably not be regarded as a definitive resolution of the overall problem. Further work on the problem is planned.
}

fourth and fifth lines, the results of two satisfactory tests of the northern hemisphere measurements are reported. Using the most precise of the five tests (see line 3 of the table), one concludes that the zero point of the $V$ magnitudes for the Hyades and Coma is established at a $2 \sigma$ level of 4 mmag.

The fifth through eighth lines of the table apply for Praesepe. This case turns out to be another one in which zero-point control does not yield a consistent set of tests. Like the color data for Praesepe and NGC 752, the $V$ data for the two clusters are on a common zero point, so it is somewhat reassuring to find that a zero-point test for NGC 752 yields satisfactory results (see the penultimate line of Table 8). However, the balance of evidence is invoked here only as a stopgap measure. Future Sturch exercises in which Praesepe is compared to nearby clusters are planned.

In Table 9, results of zero-point tests of the augmented M67 V magnitudes are listed. Extrinsic data sets from photomultipliers are used for this purpose only if a substantial fraction of the data are from more than one measurement per datum. We adopt this policy because, in our experience, photomultiplier magnitudes from a single night of observing are less likely to be reliable than photomultiplier colors obtained in the same way.

The first line of entries in Table 9 is for a data set that, like ours, has been corrected for a gradient error (see Fig. 6 of Laugalys et al. 2004). No dependence on position in the cluster is detected in the differences between the two data sets (see the fourth entry of Table 1). However, such a dependence is found for the measurements of both Sandquist and Montgomery et al. (see the second and third lines in Table 9 and the fifth and sixth lines in Table 1). This deduction confirms results obtained graphically by Laugalys et al. (see their Figs. 6 and 9). Here as in the case of $(V-I)_{\mathrm{C}}$, a small rms error is found for the Sandquist data, while a relatively large one is found for the data of Montgomery et al. (see the upper boldface entries in Table 9). With these results and the results of the $(V-I)_{\mathrm{C}}$ analysis in hand, the dubious quality of the Montgomery et al. $V I_{\mathrm{C}}$ data becomes fully evident.

The fourth and fifth lines of Table 9 show that two consistency tests of our $V$ magnitudes are satisfactory. All of the remaining 
TABLE 8

Hyades, Coma, Praesepe, NGC 752: $V$ Offsets

\begin{tabular}{|c|c|c|c|c|}
\hline Cluster & Extrinsic Source $^{\mathrm{a}}$ & Tested Data ${ }^{b}$ & $n^{\mathrm{c}}$ & $\begin{array}{c}\Delta V^{\mathrm{d}} \\
(\mathrm{mmag})\end{array}$ \\
\hline Hyades..... & Johnson \& Knuckles (1955) ${ }^{\mathrm{e}}$ & $1,2,3$ & 77 & $24 \pm 6$ \\
\hline Coma ......... & Johnson \& Knuckles $(1955)^{\mathrm{e}}$ & 1,2 & 18 & $41 \pm 8$ \\
\hline Hyades........ & Joner et al. $(2006)^{\mathrm{f}}$ & 1,2 & 29 & $-5 \pm 2$ \\
\hline Hyades, Coma... & JMIW (TJ92) & 2 & 29 & $2 \pm 3$ \\
\hline Coma & JMIW (Stetson 1991) & 1,2 & 12 & $-6 \pm 4$ \\
\hline 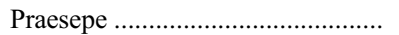 & JMIW (TJ92, JT95) ${ }^{\mathrm{g}}$ & 4 & 15 & $-20 \pm 4$ \\
\hline 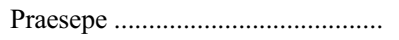 & JMIW (Dickens et al. 1968) ${ }^{\mathrm{h}}$ & 4 & 18 & $0 \pm 4$ \\
\hline 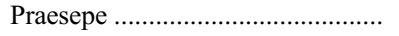 & JMIW (Stetson 1991) & 4 & 7 & $-16 \pm 4$ \\
\hline 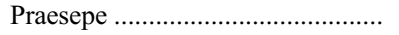 & Johnson $(1952)^{\mathrm{h}}$ & 4 & 26 & $-2 \pm 9$ \\
\hline 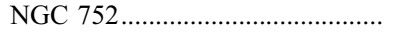 & JMIW (TJ92, JT95) ${ }^{g}$ & 4 & 14 & $-3 \pm 3$ \\
\hline 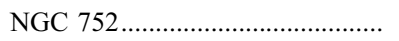 & Johnson $(1953)^{\mathrm{h}}$ & 4 & 37 & $-21 \pm 15$ \\
\hline
\end{tabular}

a JMIW is Johnson et al. (1966).

b Data are from following sources: (1) Table 4 of TJ92; (2) reductions described in $\S 3.3$ of TJ05; (3) Table 2 of Joner et al. (2006); (4)measurements described in $\S 2$.

c The quantity $n$ is the number of stars for which data are compared.

d The quantity $\Delta V$ is in the sense "extrinsic source minus this paper."

e This result supersedes a counterpart stated in $\S 2.2$ of JT90. The standard error given here includes a contribution from a zero-point error given by Johnson \& Knuckles.

${ }^{\mathrm{f}}$ Although this is a $2.5 \sigma$ result, it is not significant at $95 \%$ confidence when gauged by false-discovery rate using all entries in the table (see Miller et al. 2001).

${ }^{\mathrm{g}}$ The comparison between the JMIW data and those in this paper is made by using data from the second source listed.

${ }^{\mathrm{h}}$ The standard error given here includes a contribution from a zero-point error in the cited paper by Johnson.

entries except the one on the last line display zero-point offsets of a sort that has been well known for decades (for an early precedent, see Eggen 1963). However, the entry on the last line (see the formal offset in boldface) suggests that the $V$ zero point for the new data is modestly satisfactory. Note that the $2 \sigma$ limit is relatively large (12 mmag) in this case.

TABLE 9

M67 $V$ Magnitudes: Offrsets and Accidental Errors

\begin{tabular}{|c|c|c|c|c|}
\hline Extrinsic Source & Detector $^{\mathrm{a}}$ & $n^{\mathrm{b}}$ & $\begin{array}{c}\Delta V^{\mathrm{c}} \\
(\mathrm{mmag})\end{array}$ & $\begin{array}{c}\sigma^{\mathrm{d}} \\
(\mathrm{mmag})\end{array}$ \\
\hline Laugalys et al. (2004). & $\mathrm{CCD}$ & 202 & $24 \pm 1$ & 12 \\
\hline Sandquist (2004) ${ }^{\mathrm{e}} \ldots \ldots \ldots . . .$. & $\mathrm{CCD}$ & 73 & $\ldots$ & 9 \\
\hline C: Montgomery et al. $(1993)^{\mathrm{e}} \ldots \ldots \ldots \ldots . . . .$. & $\mathrm{CCD}$ & 175 & $\ldots$ & 30 \\
\hline 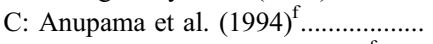 & $\mathrm{CCD}$ & 30 & $-2 \pm 3$ & 16 \\
\hline C: Chevalier \& Ilovaisky $(1991)^{\mathrm{f}} \ldots \ldots .$. & $\mathrm{CCD}$ & 29 & $-1 \pm 1$ & 5 \\
\hline Gilliland et al. $(1991)^{\mathrm{g}}$. & CCD & 63 & $17 \pm 2$ & 11 \\
\hline 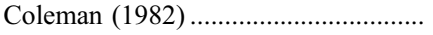 & PM & 13 & $12 \pm 3$ & 8 \\
\hline Eggen \& Sandage (1964) ..................... & PM & 56 & $22 \pm 3$ & 26 \\
\hline Sanders (1989)... & PM & 25 & $3 \pm 3$ & 18 \\
\hline JMIW (TJ92, JT97) ${ }^{\mathrm{h}}$. & PM & 10 & $4 \pm 6$ & $\cdots$ \\
\hline
\end{tabular}

${ }^{\mathrm{a}} \mathrm{PM}=$ photomultiplier data. For these data sets, only data for stars with $V<13$ are analyzed.

b The quantity $n$ is the number of stars for which data are compared.

c The quantity $\Delta V$ is in the sense "extrinsic source minus this paper."

d If numbers of measurements are given in the extrinsic source, $\sigma$ is the rms error per measurement. Otherwise, $\sigma$ is the rms error per data entry. No value of $\sigma$ is given if accidental errors are known independently for data from the extrinsic source.

e The quantity $\Delta V$ is not given because a positional correction is required instead (see Table 1).

${ }^{\mathrm{f}}$ Results in this paper have been derived (directly or indirectly) using JT90 standard-star data.

$\mathrm{g}$ We do not confirm the Laugalys et al. deduction that a positional error exists for these data.

h JMIW is Johnson et al. (1966). The comparison is made by using data on a uniform zero point from the other two cited papers.

\section{THE CONTENTS OF THE CATALOGS}

\subsection{The Catalogs and Their Photometric Sources}

With tests of data consistency complete, we describe the data files produced here and deposited in the CDS archives. As noted in $\S 1$, those files include updated color and temperature listings for the Hyades. In $\S 6$ of their paper, TJ05 present two catalogs for "effectively single" stars and one for binaries. Corresponding catalogs are produced here (for a sample, see Table 10).

When TJ05 appeared, Hyades values of $(R-I)_{\mathrm{C}}$ from Joner et al. (2006) were not yet available. Those data have now been added to the source data for the three catalogs. In addition, the catalogs for effectively single stars now include both values of $(V-K)_{\mathrm{J}}$ and $V$ magnitudes. The colors are included because they are the arguments for the temperature calibration used to derive the temperatures listed in the catalogs (see below). Users can now compare isochrones to temperatures, values of $(V-K)_{\mathrm{J}}$, and values of $(R-I)_{\mathrm{C}}$ and see whether they obtain consistent results. As for the $V$ magnitudes, they are secured (if possible) from the measurements whose zero points are tested in $\S 9$. Otherwise, data from Johnson \& Knuckles (1955) are used after the correction for the $V$ offset given in Table 8 has been applied.

The new catalogs also include two data files each for Coma, Praesepe, and NGC 752. For each cluster, one file includes either Cousins VRI photometry from TJ85 (for Coma) or the new Cousins $V R I$ data (for the other two clusters). A sample of one of those files is given in Table 11. The remaining files include temperature data and values of $V$ and $(V-K)_{\mathrm{J}}$. A sample of one of those files appears in Table 12.

For Coma, as for the Hyades, the adopted sources of $V$ data have been described in $\S 9$. For the other two clusters, new $V$ measurements (except as noted in Table 12) are reported. The reported $(V-K)_{\mathrm{J}}$ results have been transformed from values of $b-y$, $(R-I)_{\mathrm{C}}$, and $V-K_{2}$, using transformations given by TJ06. An allowance is made for the $V-K_{2}$ offset implied by the entry for data set $I_{4}$ in Table 7 . The temperatures yielded by this selection 
TABLE 10

$(R-I)_{\mathrm{C}}$ and Temperatures: Single Hyades Stars

\begin{tabular}{|c|c|c|c|c|c|c|c|c|c|c|c|}
\hline$v B$ & Cat. & Number & Kind $^{\mathrm{a}}$ & $(R-I)_{\mathrm{C}}$ & $\sigma^{\mathrm{b}}$ & $(V-K)_{\mathrm{J}}$ & $\sigma^{\mathrm{b}}$ & $\theta^{\mathrm{c}}$ & $\sigma^{\mathrm{b}}$ & $V$ & $\sigma^{\mathrm{b}}$ \\
\hline $1 \ldots \ldots \ldots \ldots \ldots \ldots \ldots$ & $\mathrm{Hic}$ & 15304 & $\mathrm{~N}$ & 0.311 & 4.0 & 1.350 & 21. & 0.846 & 4.5 & 7.376 & 3.2 \\
\hline .............. & Hic & 16529 & $\mathrm{~N}$ & 0.426 & 4.3 & 2.000 & 26. & 0.990 & 5.7 & 8.856 & 9.6 \\
\hline
\end{tabular}

a $(\mathrm{G})$ a giant; $(\mathrm{N})$ a dwarf nonbinary; (S) a binary treated as a single star.

$\mathrm{b}$ The value of $\sigma$ has been multiplied by 1000 .

${ }^{\text {c }} \theta \equiv 5040 / T_{\text {eff }}$.

of input data have standard errors that satisfy the FM standard quite adequately.

For M67, the augmented database occupies one file, while temperatures are included in another. The augmented data supersede the Joner \& Taylor (1990) $V$ magnitude for I-51, which appears to be in error. In addition, the augmented data supersede the extant data for I-198 and I-199. In this case, the data being replaced are for stars that are a little too faint for precise measurements with the system used by Joner \& Taylor. Concerning the temperature data, we note that they are based partly on values of $(V-R)_{\mathrm{C}}$ from Sandquist (2004) instead of values of $b-y$. The latter are relatively sparse for M67, so the high precision of the Sandquist colors (see Table 5) recommends them as an obvious replacement. A transformation of the Sandquist data to values of $(R-I)_{\mathrm{C}}$ is given in item 3 of Appendix A.

In the M67 temperature file, the $V$ data are averages from the augmented M67 data and those of Sandquist. Here also, the Sandquist data are adopted because their precision is high (see Table 9). Before use, they are corrected for the positional gradient noted in $\S$ 9. Those corrections are applied by using equation (1) and appropriate entries in Table 1. Besides Cousins VRI and temperature catalogs, the M67 files include one containing corrected Sandquist $V$ data. The file also includes Sandquist $(V-I)_{\mathrm{C}}$ measurements. They require no adjustment (recall $\S 6$ ) and hence have not been changed. A sample of this file is given in Table 13.

For clusters other than the Hyades, no versions of the new catalogs have been published before, and complete versions of the new catalogs are not being presented in this paper. It is therefore worthwhile to include some numerical information about the catalogs. The number of stars with Cousins photometry ranges from 17 for the sparse cluster Coma to 241 for M67. The number of stars with temperatures ranges from 27 for Coma to 347 for M67. The ranges in $V$ for stars with temperatures are about 3 mag for NGC 752, 4 mag for Coma and Praesepe, and 8 mag for M67. For values of $\theta$ ( $\left.\equiv 5040 / T_{\text {eff }}\right)$, most of the M67 rms errors range from 0.005 to 0.010 . For Coma, Praesepe, and NGC 752, the errors are somewhat smaller because the clusters are brighter. In these cases, the rms error range is from 0.001 to 0.007 .

\subsection{The Adopted Temperature Calibration}

Here, as in TJ05, temperatures are derived using the Di Benedetto (1998) calibration. Although more recent calibrations

TABLE 11

Praesepe: Cousins VRI Photometry

\begin{tabular}{ccccccccc}
\hline \hline WEBDA No. & Cat. & No. & $V$ & $\sigma^{\mathrm{a}}$ & $(V-R)_{\mathrm{C}}$ & $\sigma^{\mathrm{a}}$ & $(R-I)_{\mathrm{C}}$ & $\sigma^{\mathrm{a}}$ \\
\hline $31 \ldots \ldots \ldots \ldots \ldots \ldots .$. & KW & 31 & 9.726 & 8.0 & 0.305 & 6.2 & 0.282 & 7.4 \\
$34 \ldots \ldots \ldots \ldots \ldots \ldots .$. & KW & 34 & 9.457 & 4.6 & 0.242 & 3.9 & 0.253 & 4.2 \\
$40 \ldots \ldots \ldots \ldots \ldots \ldots \ldots$. & KW & 40 & 7.767 & 5.3 & 0.099 & 3.7 & 0.091 & 4.3 \\
\hline
\end{tabular}

${ }^{a}$ The value of $\sigma$ has been multiplied by 1000 . have been published (see, e.g., Ramírez \& Meléndez 2005), the Di Benedetto calibration is supported by an analysis performed by Taylor (2001b). The only modification made to that calibration is a response to the publication of temperatures for giants based on angular diameters (see Mozurkewich et al. 2003). When those data are compared to relation 3 in Table 4 of Di Benedetto (1998) the following zero-point correction is obtained:

$$
10^{3}\left(\Delta \log _{10} T\right)=2.1 \pm 0.9
$$

This correction is applied to the tested relation.

\subsection{Binaries}

Since the catalog data are to be used in color-magnitude analysis, policies dealing with inclusion and exclusion of binaries are required. For the Hyades, the adopted procedure is still that given in $\S 5$ of TJ05. For Coma and Praesepe, binaries are initially identified from radial velocity and speckle measurements. The papers consulted for those data include Mason et al. (1993), Abt \& Willmarth (1999), Mermilliod \& Mayor (1999), and Bouvier et al. (2001). Data for binaries identified from those papers are retained in the temperature catalogs for Coma and Praesepe only if the binaries fall within the main sequence scatter in the colormagnitude diagrams of those clusters. Photometry for binaries has not been excluded from the Cousins VRI catalogs.

M67 and NGC 752 have vertical subgiant branches, so it is difficult to use their color-magnitude diagrams to test for binary status. Fortunately, reasonably comprehensive lists of known binaries are readily available for both clusters (see Table 3 of Sandquist 2004 and Table 1 of Daniel et al. 1994, respectively). Using those lists, data for double-lined spectroscopic binaries and RS CVn stars have been excluded from the temperature catalogs. Data for single-lined binaries have been retained in those catalogs and are flagged there. Here also photometry for binaries has not been excluded from the Cousins VRI catalogs.

\subsection{Effects of Reddening Uncertainties}

It should be noted that the errors quoted for the catalog values of $\theta$ do not include the effects of the standard errors of the reddening values for the clusters. If $\delta \theta$ is defined as the error induced by the standard error of $E(B-V)$, then $\left|10^{3} \delta \theta\right|$ is $\leq 2.4$ for M67 and Praesepe, $\leq 2.0$ for NGC 752, and zero for the other clusters. At worst, these errors are comparable to the errors listed for the catalog values of $\theta$. If necessary, allowances should be made for the values of $\delta \theta$ just quoted when color-magnitude analyses are performed.

\section{PERSPECTIVES}

If we could be confident that the results in Tables 3-9 are credible prima facie, this paper could now be ended with a summary. In fact, VdBS present evidence to the contrary that cannot be overlooked. In their $\S 2.2$, those authors refer to formal 
TABLE 12

Praesepe: $V,(V-K)_{\mathrm{J}}$, Temperatures

\begin{tabular}{lcccccccc}
\hline \hline WEBDA No. & Cat. & No. & $V^{\mathrm{a}}$ & $\sigma^{\mathrm{b}}$ & $(V-K)_{\mathrm{J}}^{\mathrm{c}}$ & $\sigma^{\mathrm{b}}$ & $\theta^{\mathrm{d}}$ & $\sigma^{\mathrm{b}}$ \\
\hline $31 \ldots \ldots \ldots \ldots . . . .$. & $\mathrm{KW}$ & 31 & 9.642 & 8.0 & 1.169 & 8. & 0.806 & 1.6 \\
$34 \ldots \ldots \ldots \ldots \ldots . .$. & $\mathrm{KW}$ & 34 & 9.373 & 4.6 & 0.925 & 6. & 0.752 & 1.4 \\
$38 \ldots \ldots \ldots \ldots \ldots . .$. & $\mathrm{KW}$ & 38 & 8.616 & 9.0 & 0.673 & 15. & 0.696 & 3.2 \\
\hline
\end{tabular}

${ }^{a}$ These data have been corrected for absorption.

b The value of $\sigma$ has been multiplied by 1000 . For giants, values of $V$ are from Johnson (1952).

c These data have been corrected for reddening. For the Praesepe giants, values of $(R-I)_{\mathrm{C}}$ are averages from Canterna (1976), Mendoza (1967), and Eggen (1985). Transformations required to convert those data to $(R-I)_{\mathrm{C}}$ are from Table 4 of Taylor (1986) and Appendix B of Taylor (1996). For all giants, values of $(V-K)_{\mathrm{J}}$ are derived from $(R-I)_{\mathrm{C}}$ by applying the third transformation in Table IV of Taylor et al. (1987).

${ }^{\mathrm{d}} \theta \equiv 5040 / T_{\text {effr }}$. These data have been corrected for reddening.

zero-point offsets presented by TJ96 that are fully as small as those derived in this paper (see especially Table 3 of TJ96). The smallest offset quoted by TJ96 is $0.1 \pm 0.5 \mathrm{mmag}$, and it is featured prominently in $\S \S 7-8$ of their paper (see also the second line in Table 3 of this paper). Note that in this case, the implied $2 \sigma$ limit is $1.0 \mathrm{mmag}$. According to VdBS, however, TJ96 found no such limit that was better than about $0.01 \mathrm{mag}$ (or $10 \mathrm{mmag}$ ).

A misrepresentation of a published result by an order of magnitude is difficult to excuse. However, we leave such issues to a subsequent paper ( Taylor 2008) and focus instead on a likely reason for the problem. In a paper published at the same time as VdBS, Stetson et al. (2004) argue that for UBVRI photometry, there is a lower limit on data scatter of about 0.01-0.02 mag (see $\S 3$ of Stetson et al.). Although that view has since been modified by one of the participating authors (see Catalan et al. 2006), Stetson et al. deem the limit to be inescapable. It seems plausible that VdBS extended the limit (called here the " 10 mmag limit") to zero-point coherence and then projected it onto the results of TJ96 because they expected to see it there.

Thanks to unpublished dialog with other photometrists, we are aware of other instances in which results that satisfy the FM standard have not been accepted. Although the credibility of that standard has been addressed in print (see $\S 7.3$ of TJ06), we offer here some additional comments in the hope of clarifying the issue further.

\subsection{Zero-Point Jitter: A Published Illustration}

We first direct the attention of readers to Table 14, which is drawn from Table 1 of TJ88. That table contains $(R-I)_{\mathrm{C}}$ data vectors from measurements of M67 giants. Three available vectors of this sort have been set aside because their zero points disagree with those of the remaining data (see $\S$ IV of TJ88). Those data comprise the seven data vectors given in the table. Note that four of the vectors contain data that have been transformed from photometric systems other than $(R-I)_{\mathrm{C}}$.

The 10 mmag limit yields a definite prediction about the transformed data in particular. Since transformations of results from diverse instrumental systems to a standard system must allegedly respect the limit, the same outcome must hold a fortiori when data from one standard system are transformed to another. Note that the same conclusion is reached if one starts with the adverse judgment about transformations noted in $\S 4$. That prediction may be tested by looking first at the last two lines of Table 14, in which mean residuals and their standard errors are listed. The means are calculated relative to the data of Cohen et al. (1978).
TABLE 13

SANDQUist (2004): $(V-I)_{\mathrm{C}}$ AND CoRrected $V$

\begin{tabular}{|c|c|c|}
\hline WEBDA No. & $V^{\mathrm{a}}$ & $(V-I)_{\mathrm{C}}^{\mathrm{b}}$ \\
\hline$\ldots \ldots \ldots \ldots . .$. & 12.796 & 0.653 \\
\hline$\ldots \ldots \ldots . . .$. & 13.080 & 0.649 \\
\hline 13 & 14.115 & 0.682 \\
\hline
\end{tabular}

a These data have been corrected for positional effect.

b These data are as given by Sandquist (2004).

Note that the means do not display the predicted jitter. In addition, if the individual contributing data listed above the means are consulted, no support is found for any suspicion that the means are simply misleading representations of offsets that are actually $\geq 10$ mmag. We point out that counterpart data vectors with a similar lack of zero-point jitter are the sources of the null mean offsets quoted in $\S \S 5-6$ and $\S 8$.

The history from which Table 14 is drawn offers two other insights of interest. The table is part of a series of discussions that all present similar results (see Table VI of TJ85, Table 4 of Taylor 1986, Table 3 of T96, Tables $1-3$ and 5 of TJ05, $\S 7.3$ of TJ06, and Table 6 of T07b). There is therefore no basis for any suspicion that the examples of adherence to the FM standard given in $\delta \S 5-6$ and $\S 8$ are a dubious latter-day innovation. In addition, we note that the FM standard can apply to transformations from instrumental to standard systems, as one would expect if the standard is genuine (see Table VI of TJ85). All told, there is clearly no basis for rejecting the FM standard in favor of a universal 10 mmag limit.

\subsection{Corrective Measures: An Illustration}

We also point out that when data do not satisfy the FM standard, corrective measures may be feasible. This point may be illustrated by considering UBVRI magnitudes for NGC 188 assembled by Stetson et al. (2004). Those authors show that zeropoint differences among those data are as large as 86 mmag.

Stetson et al. (2004) analyze a total of 13 data sets. However, they do not include $B-V$ measurements by Jennens \& Helfer (1975b). The significance of the Jennens \& Helfer ( hereafter JH) data becomes clear when one notes that Jennens \& Helfer (1975b) also measured NGC 752 and that Jennens \& Helfer (1975a) report measurements of field stars. The accounts in the two papers suggest that all three sets of measurements can be regarded as the product of a de facto Sturch exercise.

Acting on this hint, we apply the $\mathrm{JH}$ data in the same way that comparable databases are used in $\S 9$. The $\mathrm{JH}$ data are compared to three data sets, with one including values of $B-V$ for NGC 188 that have been reduced by Stetson et al. (2004) to a compromise zero point. A second comparison is made to published $B-V$ measurements for NGC 752, and it reveals that the zero points of published data for that cluster cohere at the FM level (again see Table 6 of T07b). A third comparison is made to values of $B-V$ measured for field stars by JMIW. The resulting formal zeropoint difference is found to be $0.6 \pm 1.4 \mathrm{mmag}$.

Since the zero points of the JMIW and JH data are found to be closely identical, one can regard the $\mathrm{JH}$ data as a proxy for the JMIW data. A comparison of the JH and Stetson et al. data then yields a formal correction required to put the $B-V$ data of Stetson et al. (2004) on the JMIW zero point. That correction turns out to be $-11 \pm 9 \mathrm{mmag}$. The reliability of this result might be questioned because almost all of the adopted JH data for NGC 188 are from a single measurement per star. The same is true for 
TABLE 14

M67: An $(R-I)_{\mathrm{C}}$ Consistency Test

\begin{tabular}{|c|c|c|c|c|c|c|c|}
\hline Star & $\begin{array}{c}\mathrm{B}^{\mathrm{a}} \\
(R-I)_{\mathrm{K}}^{\mathrm{b}}\end{array}$ & $\begin{array}{c}\mathrm{C}^{\mathrm{c}} \\
T_{1}-T_{2}{ }^{\mathrm{b}}\end{array}$ & $\begin{array}{c}\mathrm{CFP}^{\mathrm{d}} \\
V-K^{\mathrm{b}}\end{array}$ & $\begin{array}{l}\mathrm{WMO}^{\mathrm{e}} \\
(R-I)_{\mathrm{C}}^{\mathrm{b}}\end{array}$ & $\begin{array}{c}\mathrm{KPNO}^{\mathrm{f}} \\
(R-I)_{\mathrm{C}}^{\mathrm{b}}\end{array}$ & $\begin{array}{c}\mathrm{Me}^{\mathrm{g}} \\
(R-I)_{\mathrm{J}}^{\mathrm{b}}\end{array}$ & $\begin{array}{c}\mathrm{TJ} 85^{\mathrm{h}} \\
(R-I)_{\mathrm{J}}^{\mathrm{bh}}\end{array}$ \\
\hline F $84 \ldots \ldots \ldots$ & .. & 475 & 478 & 485 & 477 & 471 & 475 \\
\hline F 105 & 544 & 548 & 541 & 554 & & 524 & \\
\hline F 108 & 617 & 606 & 609 & . & 602 & 560 & 608 \\
\hline F 141 & 470 & 468 & 476 & 475 & $\ldots$ & 471 & $\ldots$ \\
\hline F 151 & 460 & 465 & 465 & 476 & $\ldots$ & 471 & 466 \\
\hline 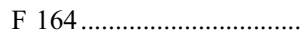 & $\ldots$ & 486 & 486 & 482 & $\ldots$ & 486 & $\ldots$ \\
\hline F 170 & 596 & $\ldots$ & 596 & 594 & $\ldots$ & 597 & 589 \\
\hline F 193 & 460 & $\ldots$ & 445 & $\ldots$ & $\ldots$ & 448 & $\ldots$ \\
\hline F 223 & & 478 & 487 & 455 & 487 & 479 & $\ldots$ \\
\hline F 224 & 491 & 486 & 481 & 489 & & 479 & $\ldots$ \\
\hline F 244 & 376 & 419 & 424 & 421 & 422 & 433 & $\ldots$ \\
\hline 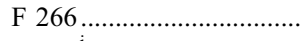 & $\ldots$ & 485 & $\ldots$ & 475 & $\ldots$ & 494 & $\cdots$ \\
\hline 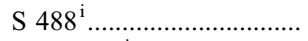 & $\ldots$ & 800 & $\ldots$ & 785 & $\ldots$ & $\ldots$ & $\ldots$ \\
\hline$\delta(R-I)_{\mathrm{C}}^{\mathrm{j}} \ldots \ldots \ldots \ldots \ldots \ldots \ldots$ & 2.9 & 1.8 & $\ldots$ & 0.3 & 2.5 & 6.3 & 2.5 \\
\hline$\sigma(\delta)^{\mathrm{k}} \ldots \ldots \ldots \ldots$ & 7.0 & 1.8 & $\ldots$ & 4.5 & 1.5 & 4.8 & 6.1 \\
\hline
\end{tabular}

${ }^{\text {a }}$ Brooke (1969). When these data are inspected, allowance should be made for the scatter introduced by their rms error of 19 mmag.

${ }^{b}$ The quoted data originally appeared as values of this color index. The Cohen et al. (1978) data appeared as values of $V-K$ on the CTIO/CIT system.

c Canterna (1976).

d Cohen et al. (1978).

e See entries for West Mountain Observatory data in Table IV of JT88.

${ }^{\mathrm{f}}$ See Table V of TJ85 and entries for KPNO data in Table IV of JT88.

${ }^{g}$ Mendoza (1967).

h See Table IV of the cited source.

${ }^{\mathrm{i}} \mathrm{S}=$ Sanders (1977).

${ }^{j}$ This is the absolute value of the mean residual from the data of Cohen et al. (1978). An error in the mean residual published originally for the WMO data has been corrected.

${ }^{\mathrm{k}}$ This is the standard error of the mean residual given just above.

the JH data for NGC 752, however, and the basic quality of those data does not appear to have been compromised as a result.

For $U-B$, this procedure is not useful because the rms error of the derived formal correction turns out to be $22 \mathrm{mmag}$. In addition, the derived $2 \sigma$ limit for the $B-V$ offset is $18 \mathrm{mmag}$, which is admittedly rather large when gauged by FM standards. Nevertheless, the size of the $B-V$ correction underscores two key points: (1) the correction process is feasible, and (2) adoption of a rigid $10 \mathrm{mmag}$ limit is undesirable partly because it is likely to discourage derivation and use of such corrections. If further Sturch comparisons are made (between NGC 188 and M67, for example), it may be quite possible to obtain even more promising correction procedures.

\section{SUMMARY}

In this paper, new Cousins VRI data are presented for NGC 752 and Praesepe, and new and extant data are combined into an augmented database for M67. For those three clusters, a set of catalogs is produced containing Cousins VRI photometry, reddening-corrected values of $(V-K)_{\mathrm{J}}$, and temperatures. The same is done for Coma by using both previously published and newly derived Cousins photometry. An extant set of catalogs for the Hyades is updated to include $V$ magnitudes and values of $(R-I)_{\mathrm{C}}$ published since the original catalogs appeared. Finally, a catalog containing Sandquist $(2004)(V-I)_{\mathrm{C}}$ and $V$ data is produced after a gradient across the face of the cluster in those $V$ data has been corrected.

In a parallel effort, the consistency of $(V-I)_{\mathrm{C}}$ databases for M67 is tested by performing an analysis with the following features:

1. the FM standard is adopted,

2. statistical analysis is applied,
3. no use of $B-V$ measurements is made,

4. no color-magnitude comparisons between M67 and NGC 188 are performed,

5. no assumption is made that independent systematic errors have canceled,

6. data reduced ultimately to the system of JT90 are included,

7. data based on standardization that is independent of ours are included,

8. results of Sturch exercises are used.

(see $\S 3$ for the first two features and $\S 4$ for the others). It is found that the new M67 data and those of Sandquist (2004) are on the $\mathrm{E}$ region zero point. In contrast, $(V-I)_{\mathrm{C}}$ measurements by Montgomery et al. (1993) (and very likely those of Richer et al. [1998] as well) are found to be too red by $27 \pm 1$ mmag or more. Zero-point tests of the Cousins VRI colors presented here for Coma, Praesepe, and NGC 752 yield satisfactory results. A likely scale factor error in the Montgomery et al. colors is found, but tests that can be performed at present suggest that the scale factors of the Sandquist colors and those presented in this paper are satisfactory. For the most part, zero-point tests of $V$ magnitudes are also satisfactory, although it is found that further work on Praesepe and M67 $V$ magnitudes would be useful.

The proper context for the results in this paper is reviewed, and it is pointed out that they follow onto a fairly extensive set of fully comparable counterparts. The need to avoid blocking the road of inquiry by arguing against the FM standard is also noted.

In the research reported in this paper, extensive use has been made of the SIMBAD database (operated at CDS, Strasbourg, France), the WEBDA database (operated at the Institute for 
Astronomy at the University of Vienna), and the Smithsonian/ NASA ADS listings. We return sincere thanks to the operators of those Web sites. Page charges for this paper have been gener- ously underwritten by the College of Physical and Mathematical Sciences and the Physics and Astronomy Department of Brigham Young University.

\section{APPENDIX A}

\section{NOTES ON TRANSFORMATIONS}

1. $(V-R)_{\mathrm{J}}$. - To transform these data to values of $(V-R)_{\mathrm{L}}$, the equations required initially are equation (5) of this paper and the equation at the top of the second page of Table 4 in Taylor (1986). In the form required here, that equation is

$$
(V-R)_{\mathrm{J}}=1.394(V-R)_{\mathrm{C}}+0.042
$$

and applies if $0.012 \mathrm{mag} \leq(V-R)_{\mathrm{C}} \leq 0.414 \mathrm{mag}$. When the two relations just mentioned have been applied, it is found that an auxiliary equation is also required. Let

$$
Y=S X+Z
$$

with $X$ being interim transformed values of $(V-R)_{\mathrm{J}}$ and $Y$ being $(V-R)_{\mathrm{L}}$. From least-squares analysis, it is found that $S=$ $1.022 \pm 0.010$ and $Z=-0.009 \pm 0.003 \mathrm{mag}$, respectively. Combining this equation with the two cited already yields the following result:

$$
(V-R)_{\mathrm{L}}=0.741(V-R)_{\mathrm{J}}-0.040
$$

It should be remembered that equation (A2) applies for the $(V-R)_{\mathrm{J}}$ data of Mendoza (1967) specifically.

2. $V-K_{2}$. - To transform these data to values of $(V-K)_{\mathrm{J}}$, equations (A13) and (A14) from Table 9 of TJ05 are revised so that $V-K_{2}$ is the independent variable and are then applied. To determine the color range for which those equations are applicable, values of $(R-I)_{\mathrm{C}}$ are transformed to $V-K_{2}$ by using the equations just noted and entries 6-8 in Table 4 of TJ06. A least-squares analysis is then applied. If $Y$ in equation (A2) represents direct values of $V-K_{2}$ and $X$ represents values of $V-K_{2}$ from transformations, it is found that $S=1.008 \pm 0.005$ and $Z=-0.015 \pm 0.008$ mag. Since neither $S-1$ nor $Z$ differs from zero at the $2 \sigma$ level, it is concluded that equations (A13) and (A14) of TJ05 are correct over the color range of the transformed data: 0.18 mag $\leq V-K_{2} \leq$ $2.97 \mathrm{mag}$. For $(R-I)_{\mathrm{C}}$, the corresponding range runs from 0.011 to $0.614 \mathrm{mag}$.

3. Sandquist values of $(V-I)_{\mathrm{C}}$. - The basic equations applied to the data of Sandquist (2004) are entries 3-5 in Table 4 of TJ06. Allowance is made for the zero-point offset listed for the Sandquist data in Table 5. When this has been done, three equations of the form

$$
(R-I)_{\mathrm{C}}=S_{S}(V-I)_{\mathrm{S}}+Z_{S}
$$

result. The derived numerical values of $S_{S}$ and $Z_{S}$ are as follows:

1. If $-0.008 \leq(V-I)_{\mathrm{S}} \leq 0.210, S_{S}=0.497$ and $Z_{S}=0.004$.

2. If $0.210 \leq(V-I)_{\mathrm{S}} \leq 0.624, S_{S}=0.479$ and $Z_{S}=0.011$.

3. If $0.624 \leq(V-I)_{\mathrm{S}} \leq 0.988, S_{S}=0.412$ and $Z_{S}=0.053$.

All values of $Z_{S}$ and all range limits are in magnitudes.

\section{APPENDIX B}

\section{COMPARISONS TO THE E REGION ZERO POINT}

The entries for the Sandquist (2004) and Montgomery et al. (1993) data in Table 5 refer those data to the zero point of the augmented database. If they are referred instead to the E region zero point, this must be done by reversing the discussion of $\S 5$ and proceeding back through the augmented M67 data and the Hyades. Here it is useful to assume that the zero-point uncertainty introduced at each of these steps is from the best of the pertinent zero-point tests in Tables 5 and 3, respectively. We also make the conservative assumption that $(V-I)_{\mathrm{C}}$ errors may be obtained by adding $(V-R)_{\mathrm{C}}$ and $(R-I)_{\mathrm{C}}$ errors in quadrature. Adding standard errors of 1.8 and $0.8 \mathrm{mmag}$ from Table 5 and 1.3 and $1.0 \mathrm{mmag}$ from Table 3 in quadrature, we find that the net "transfer error" is $2.5 \mathrm{mmag}$.

For the Montgomery et al. data, adding the transfer error in quadrature to the error quoted in Table 5 yields the following result:

$$
\Delta(V-I)_{\mathrm{C}}=27 \pm 2.8 \mathrm{mmag}
$$

Here unlike $\S 5.3, \Delta(V-I)_{\mathrm{C}}$ represents the actual difference between the Montgomery et al. data and the augmented M67 database after the 2 mmag correction discussed in $\S 5.3$ has been applied. A $t$-test shows that $\Delta(V-I)_{\mathrm{C}}$ differs from zero with $P>4.7$. No testing of this result in company with other values of $\Delta(V-I)_{\mathrm{C}}$ is required to conclude that it is highly statistically significant. 
For the Sandquist data,

$$
\Delta(V-I)_{\mathrm{C}}=-4 \pm 2.7 \text { mmag. }
$$

Here also allowance has been made for the 2 mmag correction just mentioned. In this case, $P<0$, so we conclude that the zero points of the Sandquist and E region data are indistinguishable.

\section{REFERENCES}

Abt, H., \& Willmarth, D. W. 1999, ApJ, 521, 682

An, D., Terndrup, D. M., Pinsonneault, M. H., Paulson, D. B., Hanson, R. B., \& Stauffer, J. R. 2007, ApJ, 655, 233

Anupama, G. C., Kembhavi, A, K., Prabhu, T. P., Singh, K. P., \& Bhat, P. N. 1994, A\&AS, 103, 315

Bouvier, J., Duchêne, G., Mermilliod, J.-C., \& Simon, T. 2001, A\&A, 375, 989

Brooke, A. L. 1969, Ph.D. thesis, Australian National Univ.

Buser, R. 1978, A\&A, 62, 411

Canterna, R. 1976, AJ, 81, 228

Cardelli, J. A., Clayton, G. C., \& Mathis, J. S. 1989, ApJ, 345, 245

Catalan, M., et al. 2006, ApJ, 651, L133

Chevalier, C., \& Ilovaisky, S. A. 1991, A\&AS, 90, 225

Cohen, J. G., Frogel, J. A., \& Persson, S. E. 1978, ApJ, 222, 165

Coleman, L. A. 1982, AJ, 87, 369

Crawford, D. L. 1975, AJ, 80, 955

Crawford, D. L., \& Barnes, J. V. 1969, AJ, 74, 407 . 1970, AJ, 75, 946

Crawford, D. L., \& Mandwewala, N. 1976, PASP, 88, 917

Cutri, R. M., et al. 2003, 2MASS All-Sky Catalog of Point Sources (Pasadena: IPAC)

Daniel, S. A., Latham, D. W., Mathieu, R. D., \& Twarog, B. A. 1994, PASP, 106,281

Di Benedetto, G. P. 1998, A\&A, 339, 858

Dickens, R. J., Kraft, R. P., \& Krzeminski, W. 1968, AJ, 73, 6

Eggen, O. J. 1963, ApJ, 138, 356

$$
\text { 1985, AJ, 90, } 333
$$

Eggen, O. J., \& Sandage, A. R. 1964, ApJ, 140, 130

Gilliland, R. L., et al. 1991, AJ, 101, 541

Jennens, P. A., \& Helfer, H. L. 1975a, MNRAS, 172, 667 1975b, MNRAS, 172, 681

Johnson, H. L. 1952, ApJ, 166, 640 1953, ApJ, 117, 356

Johnson, H. L., \& Knuckles, C. F. 1955, ApJ, 122, 209

Johnson, H. L., Mitchell, R. I., Iriarte, B., \& Wiśniewski, W. Z. 1966, Commun. Lunar Planet. Lab., 4, 99 (JMIW)

Joner, M. D., \& Taylor, B. J. 1988, AJ, 96, 218

1990, PASP, 102, 655 (JT90)

1995, PASP, 107, 351 (JT95)

1997, PASP, 109, 1122 (JT97)

Joner, M. D., Taylor, B. J., Laney, C. D., \& van Wyk, F. 2006, AJ, 132, 111

Landolt, A. U. 1983, AJ, 88, 439 1992, AJ, 104, 340

Laugalys, V., Kazlauskas, A., Boyle, R. P., Vrba, F. J., Davis Philip, A. G., \& Straižys, V. 2004, Baltic Astron., 13, 1
Mason, B. D., Hartkopf, W. I., McAlister, H. A., \& Sowell, J. 1993, AJ, 106, 637

Mendoza, V. E. E. 1967, Bol. Obs. Tonantzintla Tacubaya, 4, 149

Mermilliod, J.-C., \& Mayor, M. 1999, A\&A, 352, 479

Menzies, J. W., Cousins, A. W. J., Banfield, R. M., \& Laing, J. D. 1989, South African Astron. Obs. Circ., 13, 1

Miller, C. J., et al. 2001, AJ, 122, 3492

Montgomery, K. A., Marshall, L. A., \& Janes, K. A. 1993, AJ, 106, 181

Mozurkewich, D., et al. 2003, AJ, 126, 2502

Pinsonneault, M. H., Stauffer, J., Soderblom, D. R., King, J. R., \& Hanson, R. B. 1998, ApJ, 504, 170

Ramírez, I., \& Meléndez, J. 2005, ApJ, 626, 465

Richer, H., Fahlman, G. G., Rosvick, J., \& Ibata, R. 1998, ApJ, 504, L91

Sanders, W. L. 1977, A\&AS, 27, 89

. 1989, Rev. Mex. AA, 17, 31

Sandquist, E. L. 2004, MNRAS, 347, 101

Stassun, K. G., van den Berg, M., Mathieu, R. D., \& Verbunt, F. 2002, A\&A, 382,899

Stetson, P. B. 1991, AJ, 102, 589

Stetson, P. B., McClure, R. D., \& VandenBerg, D. A. 2004, PASP, 116, 1012

Strom, S. E., Strom, K. M., \& Bregman, J. N. 1971, PASP, 83, 768

Sturch, C. 1972, PASP, 84, 666 1973, PASP, 85, 724

Taylor, B. J. 1986, ApJS, 60, 577

$$
\text { . 1991, ApJS, 76, } 715
$$

. 1996, ApJS, 102, 105 (T96)

2000, A\&A, 362, 563 (T00)

2001a, A\&A, 377, 473

2001b, A\&A, 398, 721

2006, AJ, 132, 2453 ( T06)

2007a, AJ, 133, 370 ( T07a)

2007b, AJ, 134, 934 (T07b)

2008, ApJ, submitted

Taylor, B. J., \& Joner, M. D. 1985, AJ, 90, 479 (TJ85)

1988, AJ, 96, 211 (TJ88)

1992, PASP, 107, 351 ( TJ92)

1996, AJ, 111, 1338 ( TJ96)

2005, ApJS, 159, 100 (TJ05)

2006, PASP, 118, 1716 (TJ06)

Taylor, B. J., Johnson, S. B., \& Joner, M. D. 1987, AJ, 93, 1253

VandenBerg, D. A., \& Clem, J. L. 2003, AJ, 126, 778

VandenBerg, D. A., \& Stetson, P. B. 2004, PASP, 116, 997 (VdBS)

Whitford, A. E. 1958, AJ, 63, 201 